\title{
Speciation and adaptive evolution reshape antioxidant enzymatic system diversity across the phylum Nematoda
}

\author{
Lian $\mathrm{Xu}^{1,2+}$, Jian Yang ${ }^{1 \dagger}$, Meng $\mathrm{Xu}^{3 \dagger}$, Dai Shan ${ }^{4}$, Zhongdao $\mathrm{Wu}^{2^{*}}$ and Dongjuan Yuan ${ }^{5^{*}}$
}

\begin{abstract}
Background: Nematodes have evolved to survive in diverse ecological niches and can be a serious burden on agricultural economy, veterinary medicine, and public health. Antioxidant enzymes in parasitic nematodes play a critical role in defending against host oxidative stress. However, the features of the evolution of antioxidant enzymes in the phylum Nematoda remain elusive.

Results: Here, we systematically investigated the evolution and gene expression of antioxidant enzymes in the genomes of 59 nematodes and transcriptomes of 20 nematodes. Catalase has been independently lost in several orders, suggesting that it is unnecessary for some nematodes. Unlike in mammals, phospholipid hydroperoxide glutathione peroxidase is widely distributed in nematodes, among which it has evolved independently. We found that superoxide dismutase (SOD) has been present throughout nematode evolutionary process, and the extracellular isoform (SOD3) is diverged from the corresponding enzyme in mammals and has undergone duplication and differentiation in several nematodes. Moreover, the evolution of intracellular and extracellular SOD isoforms in filaria strongly indicates that extracellular SOD3 originated from intracellular SOD1 and underwent rapid evolution to form the diversity of extracellular SOD3. We identify a novel putative metal-independent extracellular SOD presenting independently in Steinernema and Strongyloididae lineage that featured a high expression level in Strongyloides larvae. Sequence divergence of SOD3 between parasitic nematodes and their closest free-living nematode, the specifically high expression in the parasitic female stage, and presence in excretory-secretory proteome of Strongyloides suggest that SOD3 may be related with parasitism.
\end{abstract}

Conclusions: This study advances our understanding of the complex evolution of antioxidant enzymes across Nematoda and provides targets for controlling parasitic nematode diseases.

Keywords: Antioxidant enzyme, Extracellular superoxide dismutase, Gene family evolution, Nematoda, Transcriptome

\footnotetext{
* Correspondence: wuzhd@mail.sysu.edu.cn; yuandj@scau.edu.cn

†Lian Xu, Jian Yang and Meng Xu contributed equally to this work.

${ }^{2}$ Department of Parasitology, Zhongshan School of Medicine, Sun Yat-sen University, Guangzhou 510080, China

${ }^{5}$ College of Veterinary Medicine, South China Agricultural University, Guangzhou 510642, China

Full list of author information is available at the end of the article
}

(c) The Author(s). 2020 Open Access This article is licensed under a Creative Commons Attribution 4.0 International License, which permits use, sharing, adaptation, distribution and reproduction in any medium or format, as long as you give appropriate credit to the original author(s) and the source, provide a link to the Creative Commons licence, and indicate if changes were made. The images or other third party material in this article are included in the article's Creative Commons licence, unless indicated otherwise in a credit line to the material. If material is not included in the article's Creative Commons licence and your intended use is not permitted by statutory regulation or exceeds the permitted use, you will need to obtain permission directly from the copyright holder. To view a copy of this licence, visit http://creativecommons.org/licenses/by/4.0/ The Creative Commons Public Domain Dedication waiver (http://creativecommons.org/publicdomain/zero/1.0/) applies to the data made available in this article, unless otherwise stated in a credit line to the data. 


\section{Background}

To date, $25 \%$ of the $\sim 23,000$ nematodes have been described to parasitize in animals and plants [1]. Nematodes are aerobic and rely on the oxygen in the atmosphere to metabolize and obtain the energy necessary for life. Low physiological concentrations of reactive oxygen species (ROS) in aerobic organisms are beneficial and involve a series of physiological activities that regulate cell differentiation, proliferation, transformation, apoptosis, protection from invading pathogens, and lifespan [2]. However, an unbalanced elevation of ROS concentration can cause damage to various biological macromolecules, such as carbohydrates, lipids, proteins, and DNA. This in turn can contribute to the development of various diseases, such as cancer, hypertension, diabetes, atherosclerosis, inflammation, and premature aging [3]. Species in the phylum Nematoda are generally large and have successfully adapted to nearly every ecosystem on Earth, from marine to fresh water and soil, from the polar region to the tropics, and from plants to animals [1]. The successful evolution of nematodes requires an effective and flexible defense system against ROS from themselves and the environment.

Nematodes have developed a complex antioxidant defense system for surviving in the environment or inside their hosts [4]. The typical antioxidant system maintains a dynamic balance in the production and decomposition of ROS in organisms through the synergistic action of superoxide dismutase (SOD), catalase (CAT), glutathione peroxidase (GPx), and peroxiredoxin (PRX) (Fig. 1a). SOD catalyzes the dismutation (or partitioning) of $\mathrm{O}_{2}^{-}$into either $\mathrm{O}_{2}$ or hydrogen peroxide $\left(\mathrm{H}_{2} \mathrm{O}_{2}\right)$. The other antioxidant enzymes, CAT, GPx, and PRX, are the major $\mathrm{H}_{2} \mathrm{O}_{2}$-detoxifying enzymes in organisms. CAT, a kind of terminal oxidase, is found in nearly all living organisms exposed to oxygen [5]. GPx functions to promote the decomposition of hydroperoxide, using glutathione as substrate, and reduces its harmful effects on the body. Loss or decrease in GPx activities in organisms can lead to a range of diseases, including diabetes, Keshan disease, and cardiovascular disease [6-8]. The deletion of phospholipid hydroperoxide glutathione peroxidase (PHGPx) causes accelerated aging and a shortened lifespan in Caenorhabditis elegans [9]. PRX is a ubiquitous family of antioxidant enzymes that controls cytokine-induced peroxide levels, and thereby mediating signal transduction in mammalian cells. The antioxidant enzymes in parasites are believed to protect them from the ROS that arise from the infection-stimulated host phagocytes [4]. However, the characterization of this antioxidant system in nematodes and its evolutionary adaption to parasitic lifestyle are not well investigated.

An increasing number of genomes of free-living and parasitic nematodes are being sequenced [10], particularly those of three major soil-transmitted nematodes, ascaris [10-13], hookworms [10, 14, 15], and whipworms [16, 17]; the vector-borne nematodes Anisakis simplex [10] (herring worm), filarial worms [10, 1821], Angiostrongylus [10, 22], and Dracunculus medinensis [10] (guinea worm); and the parthenogenetic parasite Strongyloides [23]. Extensive genomic and transcriptomic data have allowed us to explore the growth, reproduction, metabolism, and parasitism of parasites, but this has had far-reaching guiding significance for the prevention and control of parasitic diseases that can seriously harm human and animal health and economic crop growth. In this study, we focused on the genomes of 59 nematode species [10-35] from within four clades (Clades I, III, IV, and V), including free-living, and plant-parasitic, animal parasitic, and entomopathogenic nematodes. We analyzed the gene number, structure, evolution, and expression pattern of antioxidant enzymes in these nematodes, using comparative genomic and transcriptomic approaches during nematode development in free-living and parasitic nematodes in resisting oxidative damage to the worms.

\section{Results \\ Varied gene numbers of antioxidant enzymes in distinct clades of Nematoda}

A significantly smaller repertoire of antioxidant enzymes was identified in nematodes from Clade I than from other clades ( $P$ value $<0.05$, Wilcoxon rank-sum test, Additional file 1: Fig. S1). GPx and PRX varied within Clades III, IV, and V, and SOD showed the largest variation in Clade V (Fig. 1b). To understand gene number variation during nematode evolution, we inferred the phylogeny of nematodes from previous studies [10, 24, 36] and collected parasitic characters (Fig. 2). CAT was independently lost in Clade I (except the mosquito parasite, Romanomermis culicivrax) and IIIc (except the D. medinensis, Fig. 2). Nematodes had a variable gene number of SOD, and less GPx and PRX than had been found in mammals (Fig. 2). An obvious expansion of SOD could be found in some species from Clade III (e.g., Toxocara canis), Clade IV (e.g., Strongyloides papillosus), Clade Vc (e.g., Oesophagostomum dentatum), and some genera (cyst nematode Globodera in Clade IV and snailborne nematode Angiostrongylus in Clade V) (Fig. 2). Antioxidant enzymes, except for CAT, are multigene families. To further investigate the classification and evolution of antioxidant enzymes, we performed evolutionary analyses for each antioxidant enzyme.

\section{Overview of SOD gene family evolution}

SOD is the only known class of enzyme that is able to autonomously eliminate superoxide anion. Animal SOD includes three isoenzymes according to their subcellular 


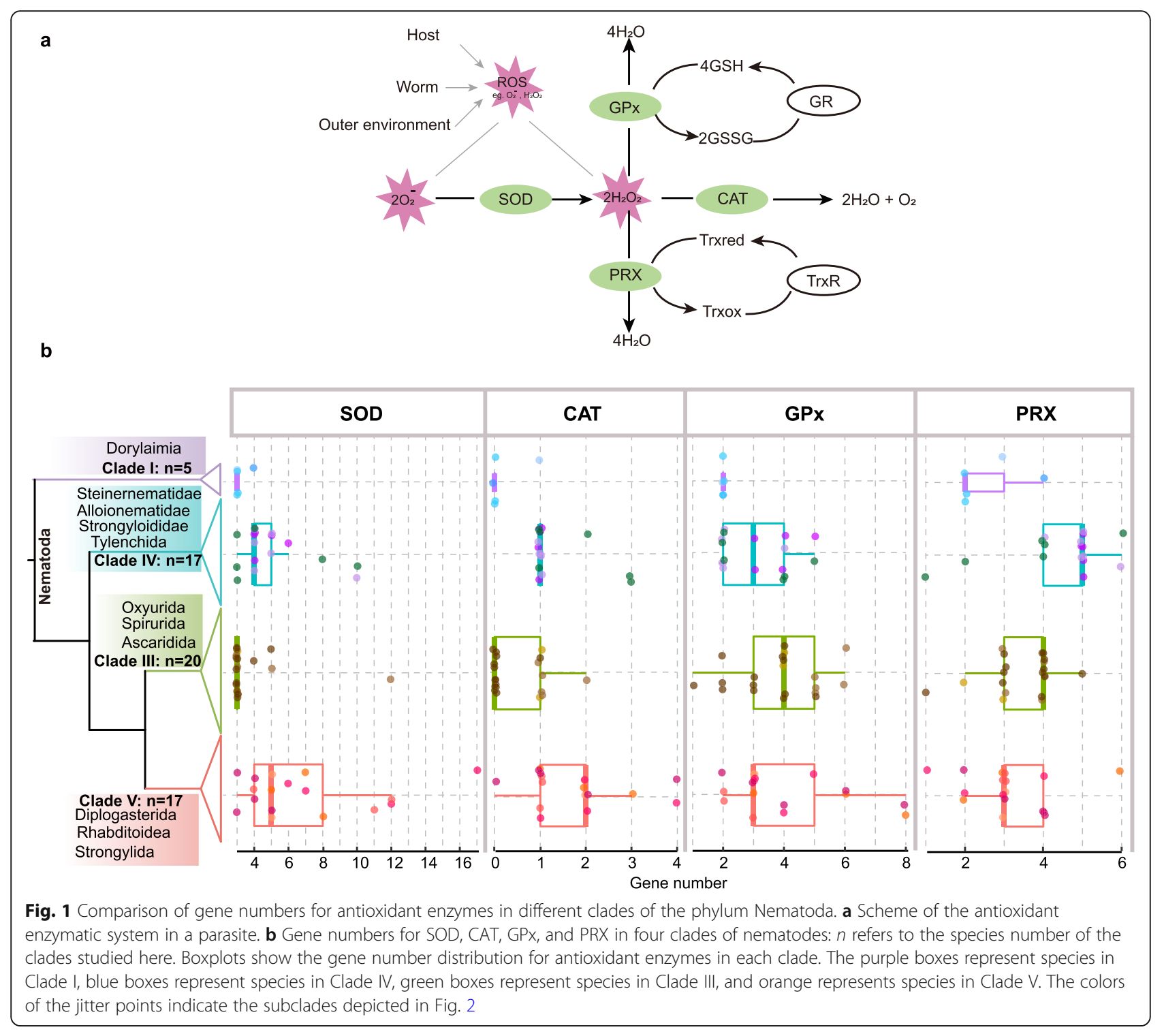

locations, namely, mitochondrial Mn-containing SOD (MnSOD or SOD2), intracellular $\mathrm{Cu} / \mathrm{Zn} \mathrm{SOD}$ (SOD1), and extracellular $\mathrm{Cu} / \mathrm{Zn}$ SOD (SOD3 or EC-SOD). Phylogenetic analyses of the SOD gene family showed three major clusters (Additional file 1: Fig. S2). To exclude a singleton that may be caused by an annotation issue, we used OrthoMCL to infer the orthologous relationship first. Nematode and mammalian SOD were clustered into 11 groups. We found that most nematode SOD1 were clustered with mammalian SOD1 into one group, except for some SOD1 from the cyst plantparasitic nematodes (Globodera). Nematode SOD2 were clustered with mammalian SOD2 into a single group. However, human and mouse SOD3 clustered together into a single group, while SOD3 from nematodes were grouped into several groups. The orthologous relationship of SOD in nematodes and mammals suggested the conservation of SOD1 and SOD2 and the divergence of SOD3 which was also supported by analyzing the phylogenetic relationship of $\mathrm{Cu} / \mathrm{Zn}$ SOD from nematodes, insects, mollusks, and vertebrates (Additional file 1: Fig. S3). To study the evolution of SOD gene family in nematodes, we performed phylogenetic analyses for each class.

In phylogenetic analyses of 61 SOD2 sequences of nematodes, SOD2 from species in Clades I, III, and V clustered together, but SOD2 from species in Clades IVa (insect-parasitic nematodes), IVb, and IVc (plant-parasitic nematodes) were not (Additional file 1: Fig. S4). Phylogenetic analyses of the SOD1 group (Fig. 3a and Additional file 1: Fig. S5) showed that some underwent extensive lineage- (plant cyst nematode Globodera [31]) and species-specific expansion (nodule nematode Oesophagostomum dentatum). SOD1 from nematodes in 


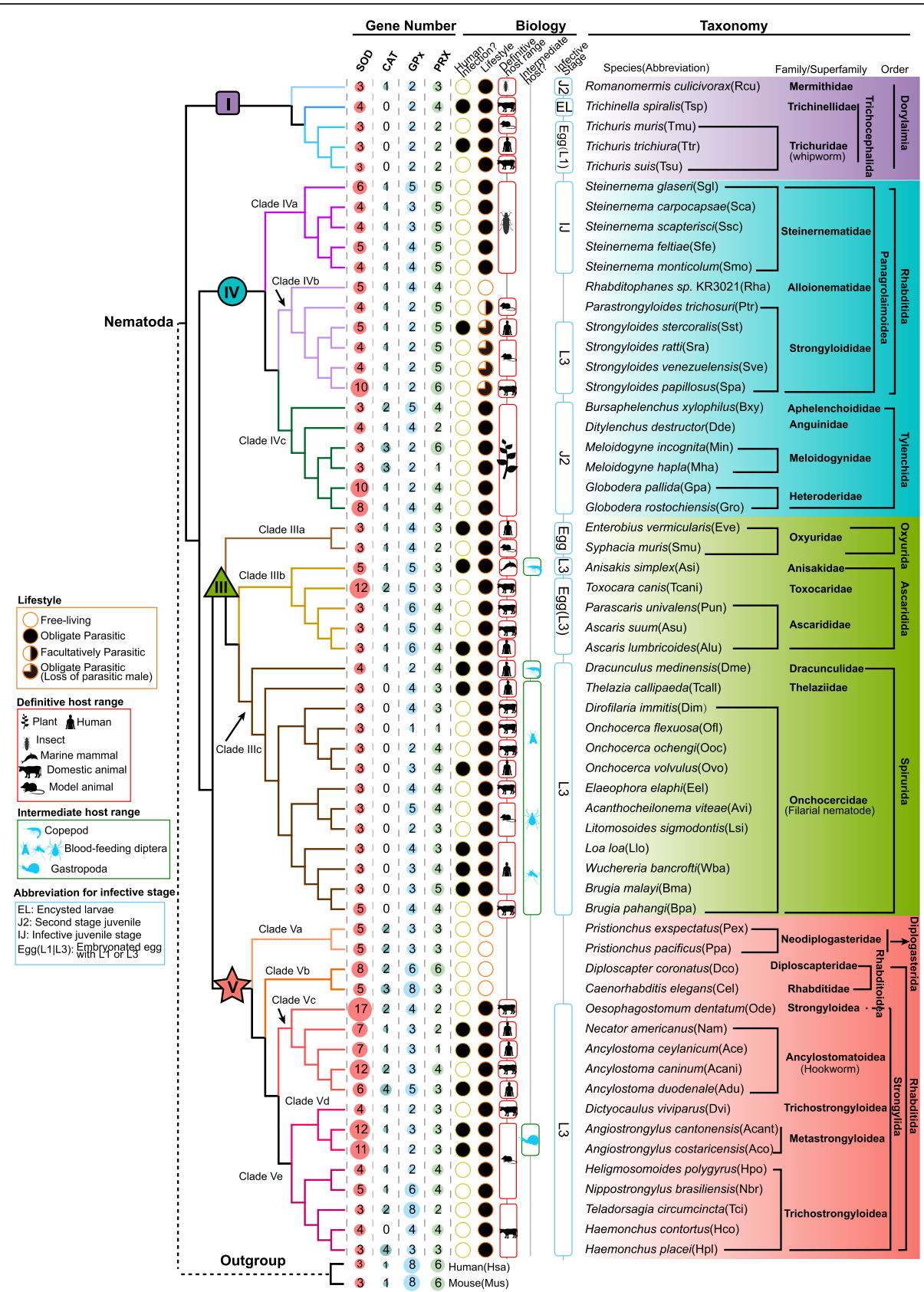

Fig. 2 Comparison of the compositions of antioxidant enzymes in 59 nematodes. The sizes of the circles represent the number of antioxidant enzymes in a category. The biology of lifestyle, human infection, definitive host range, and intermediate host range of the nematode are shown. Taxonomic classification is retrieved from the Taxonomy database. The topology of nematode phylogeny is inferred by combining previous studies $[10,24,36]$. The designated shapes for clades and colors for subclades are used consistently throughout the study

Clade III were clustered together within subclades, except for SOD1 in the guinea worm D. medinensis, but they diverged within clades. Two "isoforms" (details discussed below) were observed in filaria and Thelazia callipaeda (oriental eyeworm), but one copy was observed in other Clade III nematodes. In Clade V, SOD1 from free-living nematodes (C. elegans, D. coronatus, and Pristionchus) clustered together (cluster 1 in Fig. 3a), and
SOD1 from parasitic nematodes clustered into two groups (clusters 2 and 3 in Fig. 3a). SOD1 in cluster 3 were Clade Vc-specific and underwent extensive expansion in O. dentatum (Fig. $3 \mathrm{a}$ and Additional file 1: Fig. S5).

Phylogenetic analyses of the SOD3 group showed three major clusters, namely, nematode SOD3, mammalian SOD3, and SOD3-like (illustrated below) from 


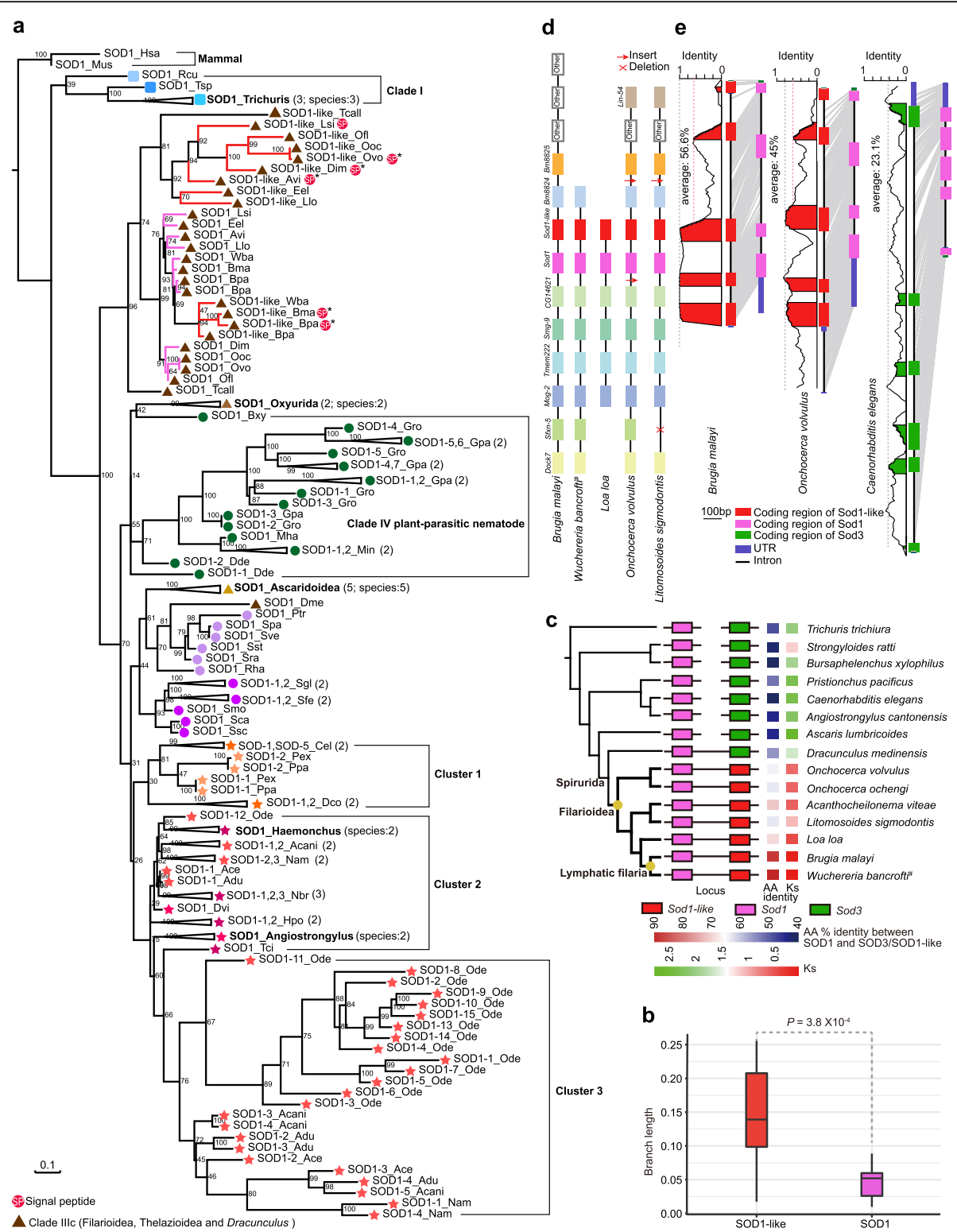

Fig. 3 Phylogenetic analyses of SOD1 or SOD1-like proteins from nematodes and mammals, and neighboring and conserved intra- and extracellular SOD isoforms in filariae. a Phylogeny of the SOD1 group in nematodes, human, and mouse. The predicted signal peptide of SOD1-like in filariae is indicated with a circled 'SP' and experimental evidence from the literature is indicated by a star. The red branch indicates SOD1-like in filaria, and the pink branch indicates SOD1 in filaria. See Additional file 1: Fig. S5 for a more detailed phylogeny without compressed nodes. The assigned shape and color indicate clades and subclades, respectively. $\mathbf{b}$ The branch length of SOD1 and SOD1-like in filaria (pink and red branches in panel a, respectively). Each genus contains multiple species, and the branch length of a gene in each species is added as their last common node. $P$ values were calculated using Student's $t$ test. c Neighboring in position and high amino acid identity between Sod1 and Sod1-like in the filariae. AA represents amino acids, and Ks is the synonymous mutation rate. A line connecting two genes indicated that they were neighboring in position. $\mathbf{d}$ Conserved gene loci around Sod1 and Sod1-like in Filarioidea with gene number more than six in the scaffold. e Conservation of Sod 1 and Sod1-like in structure and genomic sequences. Genomic identity was calculated at a 10-bp sliding window based on pairwise alignment

nematode Clades IVa and IVb (Fig. 4a and Additional file 1: Fig. S7). The lineage-specific expansion of SOD3 was observed in the genus Angiostrongylus and in T. canis. SOD3 from T. canis and A. simplex were divided into two and three branches, respectively. In one branch, SOD3 from $T$. canis and A. simplex were clustered with SOD3 from three other species of Ascaridida, suggesting that SOD3 from this branch were ancient. In the second branch (specific expansion 2 in Fig. 4a), SOD3 from $T$. canis and A. simplex were clustered into a separated branch. Considering the phylogenetic relationship and different life cycles between $T$. 


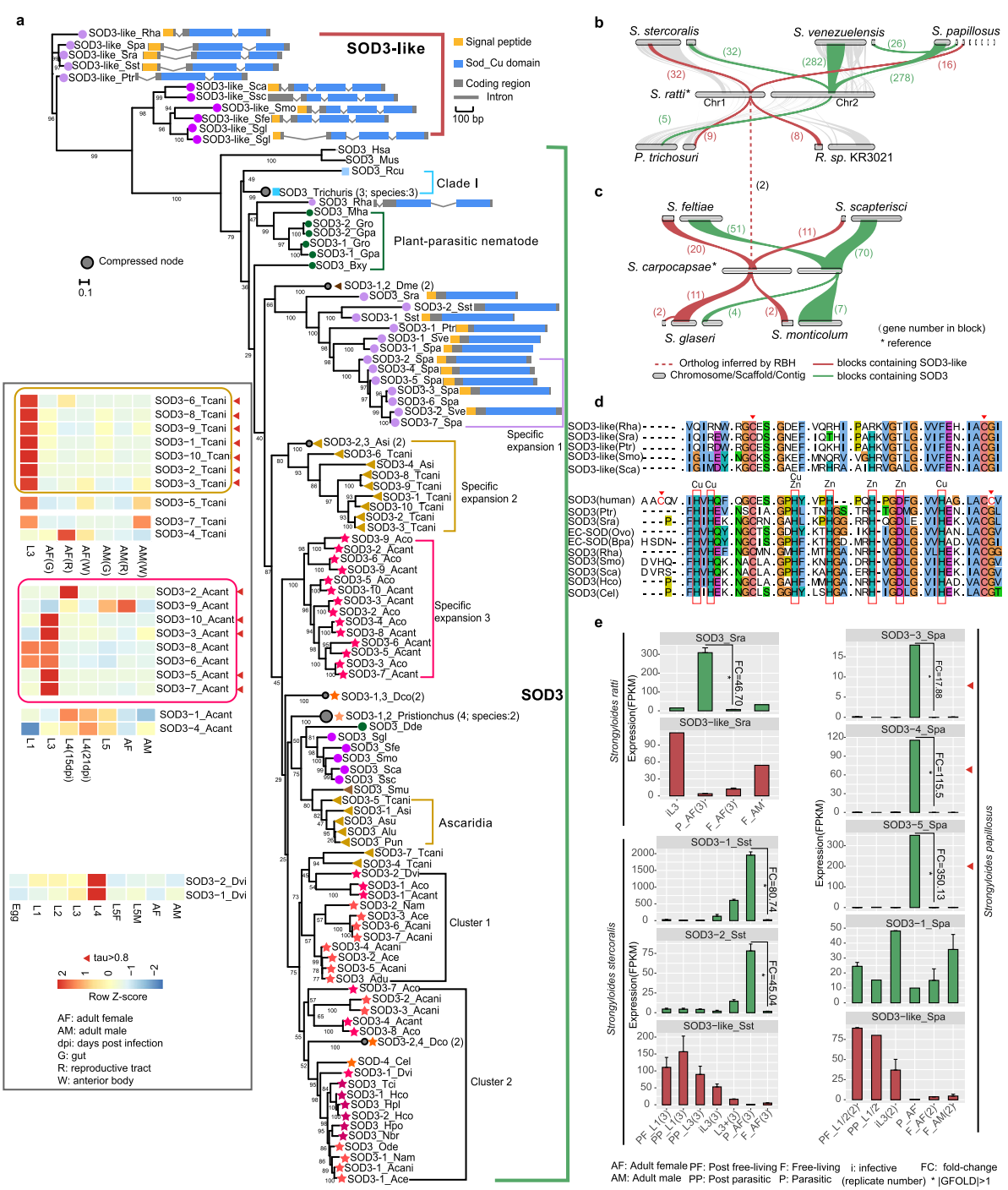

Fig. 4 Evolution of SOD3 and SOD3-like genes in nematodes, human, and mouse. a Phylogeny of SOD3 and SOD3-like genes and gene expression of SOD3 across developmental stages in three nematodes with available RNA-seq data. Lineage-specific expansion in the genus Angiostrongylus, T. canis, and S. papillosus was highlighted. The gene structures of SOD3-like in Clade IVa and Clade IVb, and SOD3 in Clade IVb are shown. Shapes and colors indicate different clades and subclades, as depicted in Fig. 2. Bootstrap values are shown in the node. Scale bar represents the number of amino acid substitutions per site. b, c Syntenic blocks in chromosomes or scaffolds or contigs containing SOD3 or SOD3-like in species from Clade IVa and Clade IVb. Numbers in the parentheses are gene number in blocks. $\mathbf{d}$ Fragments of multiple sequence alignments of EC-SOD and SOD3-like. Metal-binding sites are shown in the red box. Red triangles show disulfide bonds. Dots are used to separate blocks. Full alignment is shown in Additional file 1: Fig. S11. e Gene expression patterns of SOD3 or SOD3-like genes in Strongyloides ratti (Sra), S. stercoralis (Sst), and S. papillosus (Spa). Genes with row maximum expression (FPKM) less than 10 were not shown. Detailed expression values were shown in Additional file 6: Table S6. FPKM less than 1 was set 1 to calculate fold change. GFOLD value was calculated between freeliving adult female (F_AF) and parasitic adult female (P_AF)

canis and A. simplex, the SOD3 of specific expansion 2 in these two species showed possibly independent duplication and divergence. SOD3 in the genus Angiostrongylus underwent extensive expansion (9-10) in their last common ancestor and were divided into three clusters, one clustered with SOD3 from other Clade V nematodes (cluster 2 in Fig. 4a), one clustered with hookworm (cluster 1 in Fig. 4a), and the last being lineage-specific (specific expansion 3 in Fig. 4a). Two clusters of SOD3 were also observed in hookworms and bovine lungworm Dictyocaulus viviparus (Fig. 4a). Available public RNAseq datasets $[13,22]$ showed expression specificity (tissue specificity index $\tau \geq 0.8$ ) at a certain stage of most lineage-expansion of SOD3 isoforms in T. canis (seven out of seven) and $A$. cantonensis (five out of eight) (Fig. 4a, Additional file 6: Table S6). 
SOD3 possibly originated from SOD1, supported by the presence of extracellular SOD1-like in filaria

Interestingly, we found seven SODs with signal peptide clustered with intracellular isoform in filaria (Fig. 3a), five of which were confirmed with experimental evidence [37-39]. It should be admitted that typical SOD3 is extracellular, with signal peptide, and typical SOD1 is intracellular, without signal peptide. We called the branch that contained these seven SOD1 genes with signal peptide as extracellular SOD1-like. We also found SOD1 and SOD1-like instances in lymphatic filaria clustered together with SOD1 from other filariae, such as Onchocerca volvulus (Fig. 3a). Further, the branch length of the SOD1-like group was significantly longer than the SOD1 group (Student's $t$ test, $P=3.8 \times 10^{-4}$, Fig. 3b), suggesting a higher rate of protein evolution. We found that SOD1 and SOD1-like were neighboring in gene position in the order Spirurida, but in other nematodes, including well-studied C. elegans, intra- and extracellular SOD were not, even distributed in different scaffolds or chromosomes (Fig. 3c, the position of intra- and extracellular SOD loci in additional nematodes is shown in Additional file 1: Fig. S6). Further, the identity of amino acids found in SOD1 and SOD1-like was the highest (89-92\%) in lymphatic filaria, 63-71\% in other filariae, and only $41-55 \%$ in other nematodes. In addition, low Ks (synonymous substitutions per synonymous site) values (0.07-0.65, except for rodent filaria Litomosoides sigmodontis) between intra- and extracellular SOD1 (Fig. 3c) suggested that these genes had recently duplicated and diverged.

We also examined the synteny of chromosomes or scaffolds containing SOD1 and SOD1-like in filaria (gene number in a scaffold more than six genes were considered; information of fragment scaffolds/contigs encoding SOD1 or SOD1-like was listed in Additional file 1: Table S1). We found that this was conserved upstream of SOD1 but not downstream of extracellular SOD1-like (Fig. 3d). Then, we evaluated the genomic conservation of SOD1 and SOD1-like. The average identity of SOD1 and SOD1-like in filaria (45-55.6\%) was higher than SOD1 and SOD3 in C. elegans (23.1\%, Fig. 3e). Specifically, almost identical regions were found at the 3 ' end of the SOD1 and SOD1-like in B. malayi, and a high identity (about $80 \%$ ) was shown in three coding regions of the SOD1 and SOD1-like 3' end in O. volvulus. The identity of SOD1 and SOD1-like $5^{\prime}$ end was similar (around 60\%) in B. malayi and O. volvulus. However, there was only at most $40 \%$ identity throughout the whole region of SOD1 and SOD3 in C. elegans, and no obvious difference was found between the coding and noncoding regions or the $5^{\prime}$ end and $3^{\prime}$ end (Fig. 3e). This result strengthened the possibility that the extracellular isoform originated from the intracellular isoform by duplication and underwent genetic variation first at the $5^{\prime}$ end, and then at the noncoding region of the $3^{\prime}$ end, and last throughout the whole region, forming obviously divergent intra- and extracellular isoforms in the nematodes.

\section{SOD3 is possibly related with parasitism in Strongyloides}

SOD3 in species from Clade IVb showed sequence divergence and varied in gene number between free-living Rhabditophanes and parasitic Strongyloididae. SOD3 of $S$. papillosus underwent extensive expansion (seven copies) and duplication (two copies) in S. stercoralis (Fig. 4a). Synteny analyses showed that gene order in the syntenic block containing SOD3 was highly conserved in Strongyloides, with small-scale synteny between $S$. ratti and $P$. trichosuri, but lack synteny between $S$. ratti and Rhabditophanes (Fig. 4b). SOD3 was also identified and conserved in Steinernema, but it diverged from SOD3 in Clade IVb (Fig. 4a, c). The average CDS (coding sequence) number $(2.49 \pm 1.64)$ of whole protein-coding genes in Clade IVb was significantly less than other clades or subclades (Wilcox test, $P<0.001$, Additional file 1: Fig. S8). It has been shown that substantial intron losses occurred before the evolution of the Rhabditophanes-Parastrongyloides-Strongyloides clade [23]. SOD3 in Strongyloididae was intronless, suggesting that complete intron loss of SOD3 occurred in the last common ancestor of Strongyloididae, but has three CDSs in free-living Rhabditophanes that has close relationship with Strongyloididae (Fig. 4a, Additional file 1: Figs. S9 and S10). These evidences suggested that SOD3 in freeliving Rhabditophanes and parasitic Strongyloididae have been diverged.

A striking biology of the genus Strongyloides is femaleonly parasitic lifestyle. RNA-seq data, including parasitic adult female (simplify as P_AF) and free-living adult female (simplify as F_AF) stages in public database [23, 40, 41], enable us to deeply investigate potential roles of genes in nematode biology. Analysis of transcriptome data of three Strongyloides species showed that SOD3 were coordinately significant upregulation in P_AF compared with F_AF with fold change of 46.7 in S. ratti, 45 and 81 in S. stercoralis (two copies), and 18 350 in S. papillosus (three out of four relatively high expression of SOD3) $(\mid$ GFOLD $\mid>1$, Fig. 4e). Further, somatic proteomes of parasitic and free-living females of $S$. ratti also show that SOD3 (original gene id: SRAE_2000310200) is significantly upregulated in parasitic females compared with free-living females with fold change of 16.5 [23], which is accordant with the result of transcriptional level. In addition, SOD3 is also detected in excretorysecretory (ES) proteome of S. ratti [23, 42], suggesting its importance in parasite-host interaction. Thus, considering sequence divergence of SOD3 between free-living 
and parasitic nematodes in Clade IVb, and extremely high expression in parasitic female stages, and presence in ES, we propose that at least some copies of SOD3 in Strongyloides may be beneficial for its parasitism (details see Additional file 1: Fig. S13).

\section{Putative novel metal-independent extracellular SOD isoform (SOD3-like) independently arose in Steinernema and Clade IVb}

We also found a group of "SOD3" in species from Clade $\mathrm{IVb}$ (Strongyloides and others) and Steinernema that diverged from mammalian and nematode SOD3. Moreover, most of them encoded a signal peptide (Fig. 4a). Thus, we called the genes in this group as SOD3-like. Low amino acid identity (an average of 30\%) was observed between SOD3 and SOD3-like. A local synteny of the gene order in genomic region containing SOD3-like was conserved in Clades IVa and IVb, respectively (Fig. 4b, c). No syntenic block was detected between $S$. ratti (Clade IVb) and S. scapocapsae (Clade IVa), with the exception of two orthologous pairs (Fig. 4b, c). The branch length of SOD3-like in Steinernema was longer than that in Strongyloides, suggesting a rapid divergence of SOD3-like in Steinernema (Fig. 4a). Thus, we proposed that SOD3-like might independently occur and have undergone rapid evolution in Steinernema. The alignment of amino acids for SOD3-like and SOD3 in nematodes and human showed that SOD3-like lacked key metal-binding residues (Fig. $4 \mathrm{~d}$ and Additional file 1: Fig. S11). A 3D structural model of human and barber's pole worm (Haemonchus contortus) SOD3, and S. ratti SOD3 and SOD3-like also showed that SOD3-like could not bind with $\mathrm{Cu}$ or $\mathrm{Zn}$ (Additional file 1: Fig. S12). Unlike SOD3 in Strongyloididae species, the CDS number in SOD3-like Strongyloididae species was multiple (three CDSs, Additional file 1: Figs. S9 and S10) and with a high identity (amino acid identity 69\%) between Rhabditophanes and Strongyloididae. The transcriptomes of three Strongyloides species showed coordinately high expression of SOD3-like in the larval stages (e.g., L1/2), while low expression in the adult stage (Fig. 4e). This suggests a functional putative metal-independent extracellular SOD in Strongyloides, which requires further experiments to investigate its activity and stability.

\section{Loss, lineage-specific expansion, and duplication of CAT in several nematodes}

CAT was independently lost in species from Trichocephalida (Clade I), filaria, and T. callipaeda in Clade III, but present in their closest relatives ( $R$. culicivorax in Clade I and D. medinensis in Clade III). Phylogenic investigation showed that 62 nematode orthologs of CAT were conserved within subclades but diverged within clades (Additional file 1: Fig. S14A). In plant-parasitic nematodes, one CAT of root-knot nematode (M. hapla) clustered with CATs of other plant-parasitic nematodes, while the other five CATs of two root-knot nematodes clustered into a single cluster.

To determine whether the single cluster CAT of rootknot nematodes is lineage-specific, we analyzed other plant-parasitic nematodes [43-46], including five other root-knot nematodes. Three to eleven CATs were encoded in the draft genomes of root-knot nematodes. Phylogenetic investigation (Additional file 1: Fig. S14B) supported the contention that the specific CAT occurred and diverged in their last common ancestor. The reproduction mode in root-knot nematode is complex and different from that of other plant-parasitic nematodes, in that some of them show facultative meiotic parthenogenesis (M. hapla, M. graminicola, and M. floridensis), while others are obligatory mitotic parthenogenesis $(M$. incognita, $M$. arenaria, $M$. javanica, and $M$. enterolobii), which leads to aneuploid and polyploid genomes [47]. Approximately 2:1 or more than 3:1 of CAT gene number in root-knot nematodes were observed in mitotic parthenogenesis compared to $M$. hapla (Additional file 1: Fig. S14B). We next detected conserved nematode orthologs among seven root-knot nematode genomes and estimated ortholog number ratio relative to the diploid $M$. hapla (1:1, 2:1, $\geq 3: 1$, Additional file 1: Fig. S14C and Table S2). The result showed a higher proportion of the duplicated BUSCOs (13.1-36.7\%) in four mitotic parthenogenetic species than that in three meiotic parthenogenetic species (0.4-3.0\%, Additional file 1: Table S2). Further, summarization of $2: 1$ or $3: 1$ of orthologs relative to $M$. hapla showed large (26-42\%) in four mitotic parthenogenetic species, especially in $M$. arenaria, while was rare $(\leq 5 \%)$ in two meiotic parthenogenetic species. Result was accordant with a previous study [48]. Thus, multiple copy number of CAT in each cluster in mitotic parthenogenetic root-knot nematodes possibly was the result of their genomic characterization.

Gene expression of CATs across different development stages in $M$. incognita showed two divergent expression patterns that one (Ctl-1, Ctl-3) was high expression in endophytic stages (L3, L4, and adult female) and the other (Ctl-2) showed high expression in exophytic L2 stage (infective juvenile), while both showed low expression in exophytic egg stage (Additional file 1: Fig. S14D), according to the data of Inchan et al. [49]. Two CATs encoded in pinewood nematode B. xylophilus genome showed two divergent patterns according to RNA-seq in developmental stages [50]. One (Ctl-2) showed highly expression in the L2 stage, while the other $(\mathrm{Ctl}-1)$ showed wide expression across developmental stages, including egg (Additional file 1: Fig. S14D). CAT could induce high-virulence B. xylophilus under $\mathrm{H}_{2} \mathrm{O}_{2}$-induced stress [51]. Lineage-specific expansion 
and high expression in infective or parasitic stages of CAT may benefit for root-knot nematode colonization.

\section{Abundant phospholipid hydroperoxide glutathione peroxidase (PHGPx) was a major GPx isoform in nematodes}

Mammalian GPx could be divided into seleniumcontaining proteins (GPx1-4) and nonselenium-containing proteins (GPx5-8) [52]. GPx4, also known as PHGPx, is one of the most abundant isoforms and interferes directly with hydroperoxidation of lipids [53]. In OrthoMCL clustering analyses, GPx separated into three groups, namely mammalian GPx7 and GPx8, nematode PHGPx with mammalian GPx4, and nematode non-PHGPx (termed NPHG Px here) with mammalian GPx1, 2, 3, 5, and 6.

\section{Independently duplicated and distinct divergence of PHGPX across nematode clades}

Two PHGPx have been identified in most nematodes, but only one (GPx4) has been found in mammals (Fig. 5).
Two PHGPx in Trichuris species might have been formed by tandem duplication in their last common ancestor. The expression pattern of PHGPx in T. suis and T. muris showed one (PHGPx-2) highly expressed in adult female, while (PHGPx-1) stably expressed across the development stages $[16,17]$ with a relatively high expression in adult male stage (Additional file 6: Table S6). Recently duplicated PHGPx was also found in the genus Pristionchus (Clade V, Fig. 5). Duplicated PHGPx after speciation was also detected in Steinernema (Fig. 5). Duplicated and divergent PHGPx was found in nematodes from Clades III and V (Fig. 5). PHGPx in Clade III varied in gene number (1-5) and its sequences diverged into multiple clusters (Fig. 5). Only one PHGPx was found in $D$. medinensis, but two to three in $T$. callipaeda, as well as in 11 filarial worms, dividing into two branches (branches 1 and 2 in Fig. 5). In branch 1, the lymphatic filarial worms had one PHGPx, but other non-lymphatic filarial nematodes had two PHGPx, which were separated into two subbranches. It may be simply

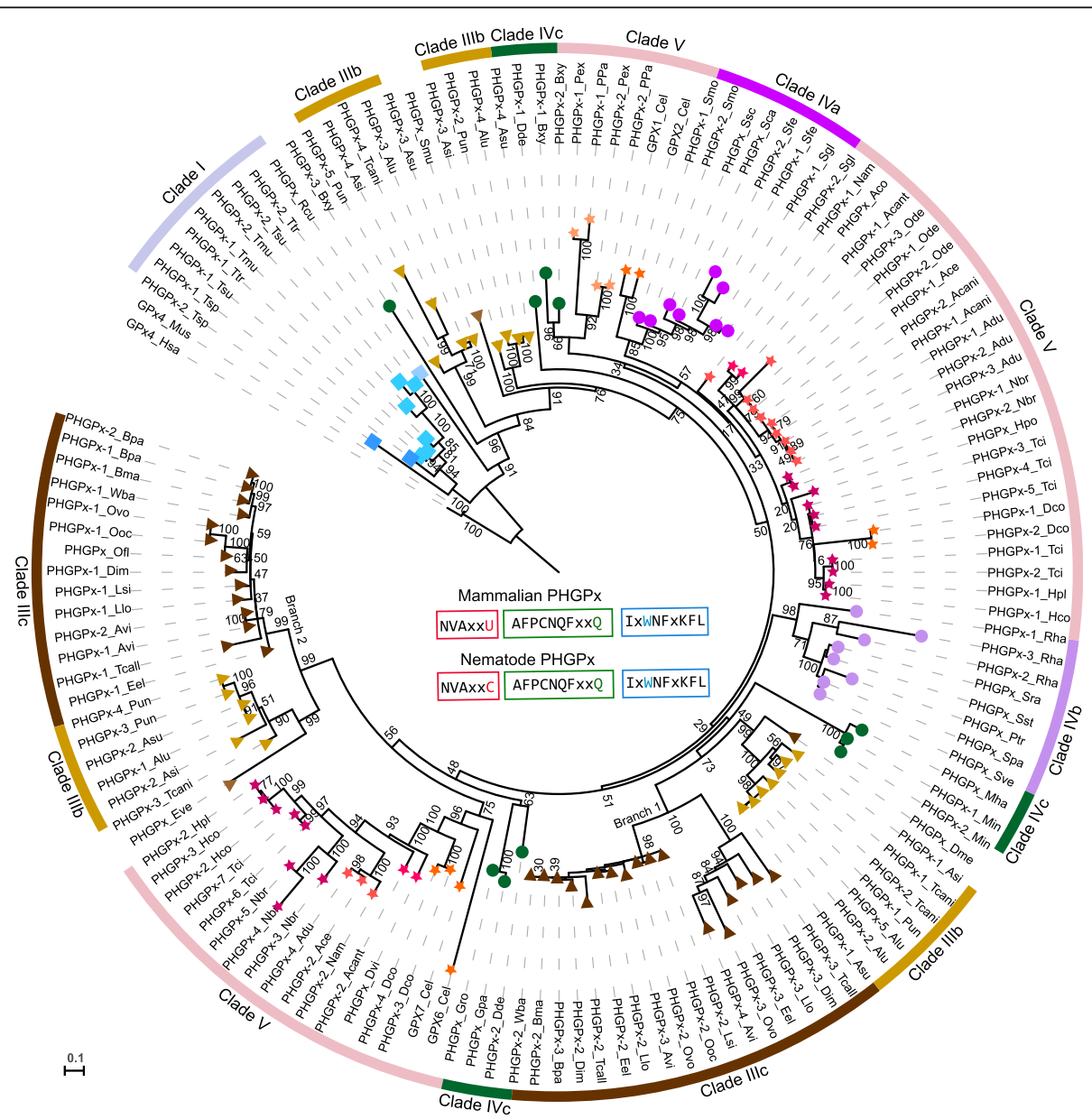

Fig. 5 Phylogenetic analyses of nematode and mammalian PHGPx. PHGPx from mammals was used as outgroup. Shape and color leaf decoration indicated clades and subclades, respectively, as depicted in Fig. 2. Scale bar represents the number of amino acid substitutions per site 
annotation issues that PHGPx-3 was absent in the original gene annotation for $O$. volvulus, but it presented clearly in its genome with transcriptional evidence (Additional file 1: Fig. S15). These data suggested that PHGPx, in this branch of non-lymphatic filaria, might have experienced duplication and loss in the last common ancestor of lymphatic filaria. The PHGPx (at least four copies) in species from Ascaridida were divided into four branches: two of them clustered with filariae, suggestive of an origin in their last common ancestor, but two other copies might have experienced independent duplication and then diverged in the ascarid lineage. In Clade V, PHGPx were clustered into two major branches and varied in gene number in some species, except for Pristionchus (Fig. 5). PHGPx duplication has continuously proceeded, being basal, intermediary, and recent, across the phylum Nematoda.

\section{Loss and pseudogenization of NPHGPx in several nematodes}

Mammalian NPHGPx has multiple copies (GPX-1, 2, 3, 5 , and 6), while most nematodes have only one copy, with the exception of Steinemema and oxyurid nematodes (Additional file 1: Fig. S16). We found that NPHG Px was absent in Trichocephalida (Clade I) but present in $R$. culicivorax. Three NPHGPx from oxyurid nematodes were identified and separated into three branches and tandemly located in the genome, suggesting that NPHGPx were duplicated in their last common ancestor and then diverged. One NPHGPx from the oxyurid nematodes lacks triads of amino acid residues $(\mathrm{C}, \mathrm{Q}$, and $\mathrm{W}$, Additional file 1: Fig. S16), which may not act as GPx. It has been shown that $B$. malayi NPHGPx (also known as gp-29) cannot metabolize $\mathrm{H}_{2} \mathrm{O}_{2}$, and D. immitis NHGPx can metabolize a limited amount of $\mathrm{H}_{2} \mathrm{O}_{2}$, while O. volvulus NPHGPx is a pseudogene with a frameshift in sequence that lacks the catalytic triad of Cys residues [4]. A frameshift also occurred in other two NPHGPx orthologs from Onchocerca species, indicating that NPHGPx underwent pseudogenization in these species (Additional file 1: Fig. S16). Thus, although NPHGPx orthologs can be found in these nematodes, their antioxidant function might have been modified. Unlike the relationship between PHGPx from the filaria and from the ascarids, NPHGPx from ascarids did not clustered with that from filarial worms and had high expression in larvae and extremely low expression in adult, as inferred from the transcriptomes of T. canis and A. suum (Additional file 1: Fig. S16 and Additional file 6: Table S6). In Clade IV, two NPHGPx paralogs in species from Steinernema appeared before speciation and diverged into two clusters (Additional file 1: Fig. S16). Expression data showed low expression of NPHGPx-2 in Steinernema, which clustered with potential nonfunctional
NPHGPx from Oxyurida (Additional file 1: Fig. S16), while NPHGPx-1 was highly expressed in the infective stage and had extremely low expression in the eggs (Additional file 1: Fig. S16).

\section{Cytosolic Prx1 was the major $\mathrm{H}_{2} \mathrm{O}_{2}$ scavenger in PRX}

The number of conserved active cysteine residues in mammalian PRX can be classified into three groups: 1Cys PRX (Prx6), typical 2-Cys PRX (Prx1), and atypical 2-Cys PRX (Prx5) [54]. In phylogenetic analyses, nematode PRX clustered into two groups of 1-Cys (Prx6) and typical 2-Cys (Prx1) with mammalian PRX (Fig. 6a). Nematodes were found to lack an ortholog of mammalian Prdx5. Prx6 was absent in Trichocephalida (Clade I), guinea worm (Clade IIIc), and pinworm (Clade IIIa).

In the Prx1 subfamily, nematode PRX clustered with human and mouse PRDX1-4 (Fig. 6a). The orthologs could be separated into two subgroups based on phylogeny. In subgroup 1, nematode PRX clustered with mammalian PRDX3, including Prdx-3 from C. elegans (Fig. 6a). Prdx-3 from C. elegans and mammals has a major location in the mitochondrion. We observed that this group of Prx1 in Clade IVb had two copies (Prx1-1 and Prx1-2) and originated in their last common ancestor (Fig. 6a). Gene expression profiles of three Strongyloides species showed that Prx1-1 has stable expression, while the expression of Prx1-2 was low in the infective stage but with higher expression in adults (Fig. 6b). One (Prx1-3) of two divergent Strongyloides Prx1 subgroup 2 copies was shown to have high expression, while the other (Prx1-4) had low expression (Fig. 6b). Although $D$. coronatus lacked Prx1 subgroup 1, four items from Prx1 subgroup 2 were present, two of which were predicted to be localized in mitochondria (Fig. 6b). It has been shown that cytosolic Prdx-2 (Prx1 subgroup 2) from $C$. elegans is more important for protecting against $\mathrm{H}_{2} \mathrm{O}_{2}$ than Prdx-3 (Prx1 subgroup 1) and is expressed in several tissues, including the intestine [55]. In C. elegans, $\operatorname{Prdx}-2$ had higher expression than Prdx-3 in developmental stages (Fig. 6b). The transcriptome data of other parasitic nematodes also showed higher expression of Prx1 in subgroup2 than in subgroup1 (Additional file 6: Table S6). This evidence suggests the importance of cytosolic Prx1 in defending against $\mathrm{H}_{2} \mathrm{O}_{2}$.

\section{Discussion}

Nematodes are one of the most abundant groups of animals on Earth and have existed since the Palaeozoic. Nematodes have evolved variable defense system to live in diverse habitats and ecological niches in lifestyles of free-living, facultative parasitic, and obligate parasitic forms. They have also experienced independent evolution to adapt to the environment, animals or plants at least several times [1]. 


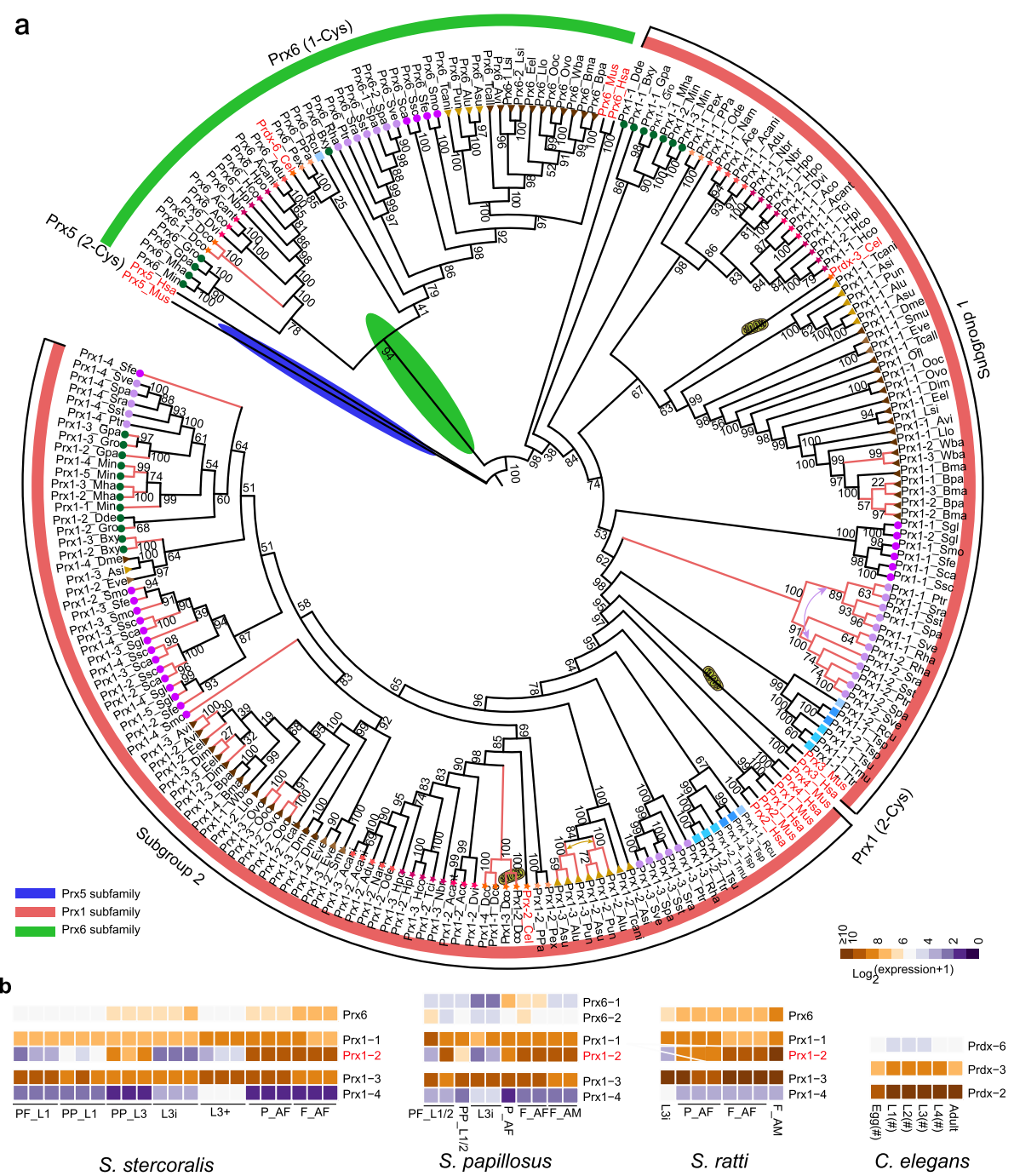

Fig. 6 Phylogenetic analyses of PRX in different nematodes and mammals. a Unrooted phylogeny of PRX in nematodes and mammals. Shape and color leaf decoration refer to clades and subclades, respectively, as depicted in Fig. 2. b Expression of PRX in four nematodes. F_ indicates free-living, $\mathrm{P}_{-}$is parasitic, AF is adult female, AM is adult male, PP is post parasitic, PF is post free-living, and i is infective. Number sign indicates RPKM

Nematodes make use of antioxidant enzymes to defend against endogenous (metabolic processes) and exogenous (host or environment) ROS [4]. However, the origin and evolution of antioxidant enzymatic system across the phylum Nematoda remain elusive. In this study, the large dataset of nematode genomes and transcriptomes enabled us to deeply investigate how antioxidant enzymes evolve to adapt diverse ecological niches.

\section{Overview of antioxidant enzyme families in nematodes and mammals}

We analyzed 294 SOD, 62 CAT, 206 GPx, and 211 PRX in 59 nematode species, and including several species from per subclade to provide more details for the evolutionary history inference of antioxidant enzymatic system in the phylum Nematoda. We also systematically classified antioxidant enzymes into several families in the phylum Nematoda based on comparative analyses and enzymatic characters. Dynamic changes in the antioxidant enzymes (SOD: SOD1, SOD2, and SOD3; CAT; GPx: PHGPx and NPHGPx; PRX: Prx1 and Prx6) were inferred by considering the phylogenies of the gene families, species, assembly, and annotation issues (change relative to the last common node is shown in Fig. 7, and detailed inferred number is shown in Additional file 1: Fig. S17). Nematodes lacked mammalian Gpx7, Gpx8, and $\operatorname{Prdx}$, and the ancestor of nematodes had a smaller number of NPHGPx (with an inferred one instance in nematodes, and five in mammals) and Prx1 (two in 


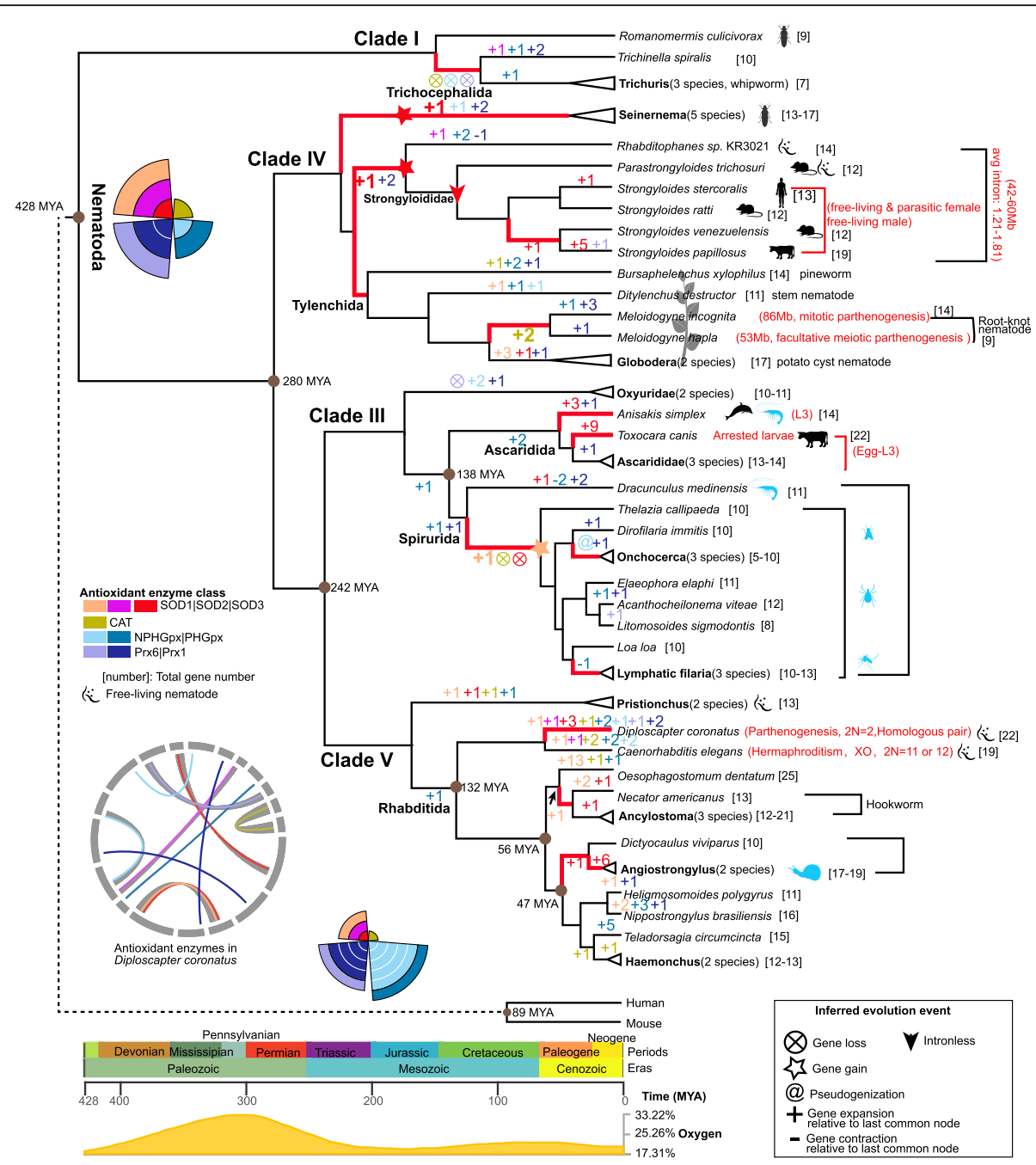

Fig. 7 Patterns of antioxidant enzyme evolution in the Nematoda. Antioxidant enzymes evolve mainly through expansion, constriction, gain and loss, and pseudogenization in nematodes. The rose diagram shows the antioxidant enzyme family numbers inferred for the ancestors of nematodes and mammals. The circos plot depicts paired antioxidant enzymes in D. coronatus. The numbers in the node represent changes in the gene family relative to the last common node. The color of the number depicts different antioxidant enzymes. The brown dot shows the estimated divergence time retrieved from the TimeTree database and our previous study. Cartoon for species parasitic definitive host or intermediate host was the same to Fig. 2. See Additional file 1: Fig. S17 for detailed antioxidant enzyme numbers for the branches. Antioxidant enzymes in nematodes with red bold branches were detailly discussed

nematodes, and four in mammals, Fig. 7 and Additional file 1: Fig. S17).

The number of antioxidant enzyme in nematodes, unlike the number in mammals, varies in different clades, specifically in Clades III, IV, and V. It has been estimated that the split between Clade III, and Clades IV and $\mathrm{V}$ was about 280 mya [22], when oxygen levels increased (oxygen data were retrieved from the TimeTree database [56], Fig. 7). We observed that CAT, Prx6, and NPHGPx were completely lost in the order Trichocephalida (Clade I) and in the families Oxyuridae, Thelaziidae, and Onchocercidae (Clade III), which suggests that these enzymes may not be essential for all of parasitic nematodes, or there may be alternative for them through other $\mathrm{H}_{2} \mathrm{O}_{2}$ scavengers. The pseudogenization of NPHG $\mathrm{Px}$ in the genus Onchocerca was also found, consistent with previous reports [4]. Contracted gene numbers of PHGPx or Prx1 were also observed in the species Rhabditophanes and $D$. medinensis, as well as in lymphatic filaria (Fig. 7). Potential novel extracellular SOD isoforms (SOD1-like and SOD3-like) were revealed using comprehensive analyses of a large array of diverse nematodes. Extensive lineage-specific expansion (5-13) of SOD1 and/or SOD3 were observed in the genus Angiostrongylus, species $S$. paillosus, $T$. canis, and $O$. dentatum (Fig. 7). Available transcriptomes showed that lineagespecific expanded genes exhibited stage-specific high expression (Fig. 4a), suggesting that at least part of these 
may be preserved by natural selection for gene dosage (SOD3 in Angiostrongylus, T. canis, and S. paillosus).

Investigation of free-living nematodes of Rhabditoidea (C. elegans and D. coronatus) showed that most of antioxidant enzyme families have multiple copies (Fig. 7). Despite the close relationship of these two species, their genomes and biology, including karyotype and reproduction, are different [57]. The duplication mechanism for antioxidant enzymes of these two nematodes differs, in that $C$. elegans features segment duplication, while $D$. coronatus shows a pattern similar to "wholegenome duplication" [58] (circos plot in Fig. 7).

\section{Crucial species nodes provide novel insights into the origin and evolution of extracellular SOD}

The origin of extracellular SOD is not clearly understood. The order Spirurida (Clade IIIc) includes the superfamilies, Chitwoodchabaudiidae (including Dracunculoidea) and Seuratidae (including Thelaziidae and Onchocercidae) [59]. The life cycles of Thelzioidea and Onchocercidae are similar to each other, in that both require a sucking arthropod as an intermediate host, and both may have arisen during the Eocene, while the life cycles found in Dracunculoidea require copepods as the intermediate host and may have arisen as early as the Triassic or the Jurassic [59]. Thus, D. medinensis, T. callipaeda, and various filaria provide an appropriate timescale to investigate this evolution. We observed that extracellular SOD isoform (SOD1-like) in T. callipaeda and 11 filarial worms clustered together with their intracellular isoform (SOD1), while the extracellular isoform (SOD3) in D. medinensis clustered together with that from other nematodes (Figs. 3a and 4a). SOD1 and SOD1-like were neighboring and conserved in the gene order in Filarioidea. Further, a different evolutionary rate (branch length, amino acid identity, and genomic identity) was observed in the comparison between SOD1 and SOD1-like and between SOD1-like from lymphatic filaria (e.g., B. malayi) and other filarial nematodes (e.g., O. volvulus). The identity between SOD1 and SOD1-like in filaria was higher than that for other nematodes (such as SOD1 and SOD3 in C. elegans) at the amino acid and genome levels. These evidences strongly support the contention that SOD1-like was duplicated from SOD1 isoform and underwent rapid evolution in filaria. Extracellular SOD in filaria also provides an excellent instance to describe its origin and evolutionary trajectory. It has been shown that extracellular SOD diverged from the intracellular isoform at an early stage of evolution, which occurred before the appearance of plants, fungi, and metazoans [60]. Thus, we speculate that the divergent extracellular SOD (SOD3) may have been presented in their last common ancestor and underwent lost, and then SOD1 recently underwent tandem duplication and diverged into extracellular SOD.

The nematodes in Clade IVa include entomopathogenic nematodes Steinernema. Nematodes in Clade IVb include taxa with diverse lifestyles, including free-living (Rhabditophane), facultative parasitic (Parastrongyloides), and obligate parasitic (Strongyloides) forms. We observed a putative novel extracellular SOD isoform (SOD3-like) that may independently appear in the Clades IVa and IVb (Fig. 7). We showed that SOD3-like lacked metal-binding sites and may not bind $\mathrm{Cu}$ and $\mathrm{Zn}$ (Fig. 4d, Additional file 1: Figs. S11 and S12). Cu/Zn SOD requires $\mathrm{Cu}$ for catalysis and $\mathrm{Zn}$ to enhance catalytic efficiency and stabilize the protein and is widespread from the periplasm of bacteria to virtually every organelle in the human cell [61]. In addition, a large amount of extracellular $\mathrm{Cu}$-only SODs have been identified in fungi [61], indicating the possibility that metal-independent extracellular SOD may also exist. Transcriptome data from three Strongyloides species and two Steinernema species showed a relatively higher expression of SOD3-like in larvae than in adults in Stronglyloides, and a relatively low expression one in Steinernema (Fig. 4e and Additional file 6: Table S6), which implies a potential functional SOD3-like in Strongyloides. Phylogenetic analyses (Fig. 4a) also showed divergent and rapid evolution of SOD3-like in Steinernema. Further functional experiments to establish the activity and stability of metal-independent SOD3-like are required. In addition to SOD3-like in Clade IVb, extracellular $\mathrm{Cu} / \mathrm{Zn}$ SOD (SOD3) is also present. A close relationship and similar gene structure were found between SOD3-like in free-living Rhabditophanes (three CDSs) and parasitic Strongyloididae (three CDSs), while the phylogenetic relationship and gene structure of SOD3 were divergent (three CDSs for Rhabditophanes, but one CDS for Strongyloididae, Additional file 1: Figs. S9 and S10). SOD3 in Strongyloididae underwent extensive intron loss, with extremely high expression in the parasitic stage. This result suggests that SOD3-like does not originate from SOD3 and experienced a slow evolutionary rate in Clade IVb. The origin of putative SOD3-like is still not understood, and more data are necessary.

\section{Evolution of antioxidant enzymes associated with adaptive evolution across the phylum Nematoda}

Adult Trichuris (whipworm) parasites in Clade I possess a specialized morphology (whip-like) that the slender anterior part (stichosome) of the body is burrowed within the intestinal epithelium of the host, while the bulbous posterior end of the body lies free in the intestinal lumen of the host, and the anterior region of the whipworm is closely contacted with host intestinal cells and immune system [16]. Transcriptome [16, 17] of adult anterior and posterior body in two Trichuris species showed 
consistently significant upregulation (GFOLD $>1$ ) of SOD3 in anterior (mixed sex) than posterior (female and male) region, with log2-transformed fold change of 2.77-2.88 and 2.85-3.22 in T. suis (without biological replicate) and T. muris (three biological replicates), respectively (Additional file 6: Table S6). This result supports the importance of SOD3 in parasite-host interaction.

In Clade IV, the striking biology of the genus Strongyloides, its female-only parasitic stage and the free-living female and male, provide insights into the genetic basis and evolution of parasitism. Evolutionary analyses of antioxidant enzymes showed that only SOD3 exhibited divergence between free-living Rhabditophanes and parasitic Strongyloididae (Fig. 4a). In details, low amino acid identity, lack synteny in gene order that contains SOD3, complete intron loss in the ancestor of parasitic Strongyloididae after diverged from the last common ancestor of Rhabditophanes-Parastrongyloides-Strongyloides clade support sequence divergence of SOD3 between free-living Rhabditophanes and parasitic Strongyloididae. Transcriptome of three Strongyloides species showed a specific high expression of some copies of SOD3 in the parasitic stage (Fig. 4a and Additional file 6: Table S6), and higher expression of SOD3 in protein level in S. ratti parasitic stage compared to free-living ones, and presence in ES of S. ratti [23], providing a strong support that SOD3 may also be related to parasitism in Strongyloides. Other antioxidant enzymes in species from Clade IVb showed consistency with the speciation and expression in Strongyloides had little difference between the free-living and parasitic stages (Additional file 6: Table S6). Taken together, we propose that SOD3 might be the major antioxidant enzyme in Strongyloididae related with its survival in the host.

The nematodes in Clade Vd include Dictyocaulidae (bovine lungworm) and Angiostrongylidae (rat lungworm and A. costaricensis). The life cycle of the genus Angiostrongylus is complex, involving a definitive host and intermediate host or paratenic host [62], while the life cycle of $D$. viviparus is simple. Our analyses showed that two copies of SOD3 existed in their last common ancestor, but the genus Angiostrongylus had lineagespecific expansion of SOD3 (at least 6 genes). Further, our previous study [63] and transcriptome data from $A$. cantonensis and $D$. viviparous showed that ancestor SOD3 had a higher expression in the mammalian stage (Fig. 4a). Localized expression of extracellular SOD3 was observed at the cuticle and around the intestine in $A$. cantonensis [63], or hypodermis localization in B. malayi and $O$. volvulus $[37,64]$. Extracellular $\mathrm{Cu} / \mathrm{Zn} \mathrm{SOD}$ activity was detected in the ES of $A$. cantonensis [65]. It can infer that extracellular $\mathrm{Cu} / \mathrm{Zn}$ SOD might be easily secreted into the extracellular matrix or ES for defense against host ROS. Lineage-specific expansion was found in Angiostrongylus and was absent in its close taxa, and relatively high expression was found in the snail-borne stage, all of which support our previous hypothesis that lineage-specific expansion of SOD3 may promote its survival in its snail host [22].

In addition, comparison of antioxidant enzyme expression in adult female from parasitic and free-living stages of three Strongyloides species, or in adult anterior and posterior body from two Trichuris species, showed most antioxidant enzymes (including SOD1, SOD2, CAT, PRX) were not significantly differential expression, except for SOD3 (Additional file 6: Table S6). It is known that $\mathrm{H}_{2} \mathrm{O}_{2}$ could be across membrane by the diffusion and aquaporin channel to perform functions, while $\mathrm{O}_{2}{ }^{-}$ is limited [66, 67]. Thus, we proposed that the functions of some antioxidant enzymes, including SOD1, SOD2, CAT, PRX, might maintain the low physiological concentrations of ROS for a series of physiological activities of nematodes.

\section{Conclusions}

In summary, we provide the first systematic annotation and classification of antioxidant enzymes for nematodes. Comparative analyses of antioxidant enzymes in the phylum Nematoda provided novel insights into the origin of extracellular SOD and its evolutionary trajectory, and it revealed a novel metal-independent putative extracellular SOD3-like. Further, the close relationship of nematodes with diverse life cycles or lifestyle also provide some evidences for the lineage-specific expansion of extracellular SOD3 related to the complex life cycles of nematodes, and extracellular SOD3 may be beneficial for parasitism. Alternative enzymes neutralizing $\mathrm{H}_{2} \mathrm{O}_{2}$ (CAT, GPx, and PRX) showed that some had been lost and that there are minor variations in their number and expression across parasitic nematodes. Our study provides a deep understanding of the evolution of antioxidant enzymes in nematodes, which could provide targets for anthelmintic control.

\section{Methods}

\section{Genome-wide identification of antioxidant enzymes}

In all, 103 nematode assemblies (86 species), not including A. cantonensis [22] and $N$. brasiliensis [34], were retrieved from Wormbase WBPS10 [68]. We filtered parasitic species with low assembly metrics (Scaffold N50 and Contig N50 $<1 \mathrm{~kb}$ ), and we retained one highquality assembly for species with multi-assemblies. We chose C. elegans and T. spiralis as representatives for the genus Caenorhabditis and Trichinella, respectively (59 species, Additional file 2: Table S3). To avoid the introduction of differences by different annotation methods from original genome annotations, we employed a 
uniform pipeline to identity antioxidant enzymes across nematode genomes, with the exception of well-studied C. elegans. This pipeline was similar to the olfactory receptor gene annotation done for the seahorse genome [69]. Simply as following: first, we downloaded known helminth antioxidant enzymes deposited in the SwissProt database as queries for baiting homologies in the nematode genomes. Then, these queries were aligned for each genome using BLAST [70] (v2.2.26) with parameters "-p tblastn -F F -e 1e-5." Solar [71] software (v0.9.6) was used to join high-score blocks between each pair of protein mapping results, and alignment rates of less than 0.5 were discarded. Subsequently, protein sequences were mapped to the genome using GeneWise [72] (wise package, v2.2.3) and extended $500 \mathrm{bp}$ upstream and downstream to define integrated gene models. The domain was predicted from a search of the Pfam database (v28.0) using the program HMMER [73] (v3.1b2) with an $e$ value less than 0.001 . Domains encoded in different antioxidant enzymes from $C$. elegans were used to further discard the fragmentary genes from other nematodes. We also used HMMER to detect potential antioxidant enzymes in the original genome annotation. Then, we manually examined sequences of these antioxidant enzymes in original gene annotation and reannotated gene annotation by checking information, such as gene length, domain annotation, and blast hit information. All antioxidant enzyme information discussed in this study is listed in Additional file 3: Table S4, and reannotated sequences of antioxidant enzymes in 58 nematodes are listed in Additional file 4. Signal peptide was inferred by Phobius [74] (v1.01). Multiple sequence alignment was edited with Jalview [75] (v2.11.0). The 3D protein structure for SOD3 (4oja as the template) and SOD3-like ( $\ln 19$ as the template) of $S$. ratti were predicted using the online SWISS-MODEL (https://swissmodel.expasy.org). The 3D structures of the protein for SOD3 from human and $H$. contortus were retrieved from SWISS-MODEL. Protein structure visualization was performed using PyMOL (http://pymol.org).

\section{Comparative genomic analysis}

Phylogenetic relationship of 59 nematodes was inferred $[10,24,36]$. Divergence times for several species was retrieved from the TimeTree database [56] (http://www. timetree.org/) and our previous estimation [22]. Oxygen content change was retrieved from the TimeTree database. To study the evolution of antioxidant enzymes across species in the phylum Nematoda, we performed phylogenetic analyses for each gene family. Corresponding genes from human and mouse were used as an outgroup, except for SOD3 and PRX. Firstly, we used MUSCLE [76] (v3.8.31) to perform multiple sequence alignment based on protein sequence. Poor alignment was trimmed with TrimAl [77] (v1.2). Then, IQ-TREE [78] (v1.6) was employed to select the best model for Maximum-Likelihood and reconstruct phylogenetic trees. Visualization were conducted using Evolview [79] (https://www.evolgenius.info/evolview) or iTOL [80] (https://itol.embl.de/). For SOD and GPx gene families, due to unclear resolve class, we first clustered them into orthologous groups using OrthoMCL to remove divergent singleton sequences [81] and then reconstructed the phylogeny for subfamilies independently. Nonsynonymous substitutions per nonsynonymous site $(K a)$ and synonymous substitutions per synonymous site $(K s)$ of pairwise pairs were calculated by Yn00 from PAML package [82] (v4.9h). Synteny of genes in different species was performed using MCSCANX [83] (https:// github.com/tanghaibao/jcvi) with default parameters. We also used reciprocal best hit (RBH) to detect orthologous genes in two genomes. To estimate ortholog copy number in seven root-knot nematode genomes, first, we employed the BUSCO pipeline [84] to detect orthologs of single-copy genes in nematodes (nematode dataset deposited in OrthoDB v10) with the parameter "-m genome --augustus_species caenorhabditis" (Additional file 1: Table S2). Then, the potential ortholog (single-copy, duplicated and fragmented BUSCO groups were all considered) copy number $(1: 1,2: 1, \geq 3: 1)$ relative to the diploid M. hapla was summarized (Additional file 1: Table S2 and Fig. S14C).

\section{Species abbreviation and antioxidant enzyme nomenclature}

The species abbreviations consisted of an uppercase initial letter from the genus name and two lowercase initial letters from the species name. If repeated species abbreviations occurred, four lowercase initial letters from the species name were extracted. The newly designated gene names were represented by the name of the antioxidant enzymes family with an underline and three or five letters species abbreviation (e.g. Sod2_Acant for Sod2 from A. cantonensis). For multiple gene duplicates, each copy was designated by a dash and a number (e.g., Sod3-1_ Acant, Sod3-2_Acant, and Sod3-3_Acant). To avoid confusion and conflict, gene names for C. elegans and outgroup (human and mouse) were kept.

\section{Antioxidant enzyme expression profile analyses}

To investigate gene expression pattern of these antioxidant enzymes during the nematode development, we downloaded RNA-seq datasets of the developmental stages from 20 nematodes with data available in SRA database (Additional file 5: Table S5). FastQC (v0.11) was used to check the quality, and Trimmomatic [85] (v0.38) was used to filter low-quality reads. We then aligned reads to the reference genome with HISAT2 [86] 
(v2.1), and alignment summary information is listed in Additional file 5: Table S5. We used featureCount from Subread package [87] (v1.6) to obtain read count and normalized sequencing depth. FPKM or RPKM was used to normalize expression for paired-end or single-end RNA-seq, respectively. Pearson correlation of samples (or biological replicates) in a species is shown in Additional file 5: Table S5. Hierarchical clustering analysis of gene expression profile was conducted by Pheatmap of $R$ package using "Euclidean distance" as clustering distance method. Gene expression specificity [88] was calculated based on normalized log-transformed expression values across all available data sets. Differential expression analysis was only performed on samples with at least two biological replicates or nematodes with at least two species (available RNA-seq data) in a genus and with sufficient data amount ( $>10$ million reads) and appropriate overall genome alignment $(>70 \%)$. To detect differential expressed antioxidant enzymes among nematode transcriptome data, we employed GFOLD [89] (v1.1.4), a tool for ranking differentially expressed genes from RNA-seq data, which is specifically useful when no replicate is available. Genes with $\mid$ GFOLD $\mid>1$ were defined as significantly differentially expressed (blue sheet in Additional file 6: Table S6).

\section{Supplementary information}

Supplementary information accompanies this paper at https://doi.org/10 1186/s12915-020-00896-z.

Additional file 1: Supplementary Figs. S1 to S17, and Tables S1-S2.

Additional file 2: Table S3. Assembly and gene annotation information of 59 nematodes.

Additional file 3: Table S4. Gene information of antioxidant enzymes in reannotation and original gene annotation in 59 nematodes.

Additional file 4:. Protein-coding transcript (cds) and Protein-coding transcript translation (pep) sequences, and GFF ( $\mathrm{gff}$ ) files of antioxidant enzymes in 58 nematodes.

Additional file 5: Table S5. Transcriptome data information (SRA information and genome alignment summary) for 20 nematodes.

Additional file 6: Table S6. Expression of antioxidant enzymes in 20 nematode developmental stages and pairwise comparison of antioxidant enzymes in 11 nematodes (blue sheets) detected by GFOLD.

\section{Abbreviations}

ROS: Reactive oxygen species; SOD: Superoxide dismutase; CAT: Catalase; GPx: Glutathione peroxidase; PRX: Peroxiredoxin; PHGPx: Phospholipid hydroperoxide glutathione peroxidase; ES: Excretory-secretory; FPKM: Fragments Per Kilobase of transcript per Million mapped reads; RPKM: Reads per kilobase of gene per million mapped reads

\section{Acknowledgements}

We are grateful to Dr. Xuanmin Guang (BGl-Shenzhen) for his comments and suggestions on the manuscript.

\section{Authors' contributions}

D.Y. and L.X. supervised the whole project. L.X., J.Y., and M.X. performed the major research and wrote the manuscript in equal contribution. Z.W. provided his professional expertise. The authors read and approved the final manuscript.

\section{Funding}

This work was supported by the National Natural Science Foundation of China (32072881); Natural Science Foundation of Guangdong Province, China (2020A1515010294); National Research and Development Plan of China (2016YFC1200500); Science and Technology Program of Guangzhou, China (201804010006); and Innovation Team Project of Guangdong University, China (2019KCXTD001).

\section{Availability of data and materials}

The datasets analyzed during the current study are available in the public databases (Wormbase and NCBI). All relevant accessions of genomes and transcriptomes are listed in Additional file 2: Table S3 and Additional file 5: Table S5.

Ethics approval and consent to participate

Not applicable

\section{Consent for publication}

Not applicable

\section{Competing interests}

The authors declare no conflict of interest.

\section{Author details}

${ }^{1}$ Key Laboratory of Neuroregeneration, Ministry of Education and Jiangsu Province, Co-innovation Center of Neuroregeneration, Nantong University, Nantong 226001, China. ${ }^{2}$ Department of Parasitology, Zhongshan School of Medicine, Sun Yat-sen University, Guangzhou 510080, China. ${ }^{3}$ Department of Ecology, Jinan University, Guangzhou 510632, China. ${ }^{4}$ BGI Genomics, BGI-Shenzhen, Shenzhen 518083, China. ${ }^{5}$ College of Veterinary Medicine, South China Agricultural University, Guangzhou 510642, China.

Received: 18 February 2020 Accepted: 12 October 2020

Published online: 26 November 2020

\section{References}

1. Blaxter M, Koutsovoulos G. The evolution of parasitism in Nematoda. Parasitology. 2015;142(Suppl 1):S26-39.

2. Bazopoulou D, Knoefler D, Zheng Y, Ulrich K, Oleson BJ, Xie L, Kim M, Kaufmann A, Lee YT, Dou Y, et al. Developmental ROS individualizes organismal stress resistance and lifespan. Nature. 2019;576(7786):301-5.

3. Schieber M, Chandel NS. ROS function in redox signaling and oxidative stress. Curr Biol. 2014;24(10):R453-62.

4. Henkle-Duhrsen K, Kampkotter A. Antioxidant enzyme families in parasitic nematodes. Mol Biochem Parasitol. 2001;114(2):129-42.

5. Zamocky M, Furtmuller PG, Obinger C. Evolution of catalases from bacteria to humans. Antioxid Redox Signal. 2008;10(9):1527-48.

6. Buijsse B, Lee DH, Steffen L, Erickson RR, Luepker RV, Jacobs DR Jr, Holtzman J. Low serum glutathione peroxidase activity is associated with increased cardiovascular mortality in individuals with low HDLc's. PLoS One. 2012;7(6): e38901.

7. Goyal R, Singhai M, Faizy AF. Glutathione peroxidase activity in obese and nonobese diabetic patients and role of hyperglycemia in oxidative stress. J Midlife Health. 2011;2(2):72-6.

8. Thomson CD. Assessment of requirements for selenium and adequacy of selenium status: a review. Eur J Clin Nutr. 2004;58(3):391-402.

9. Sakamoto T, Maebayashi K, Nakagawa Y, Imai H. Deletion of the four phospholipid hydroperoxide glutathione peroxidase genes accelerates aging in Caenorhabditis elegans. Genes Cells. 2014;19(10):778-92.

10. International Helminth Genomes Consortium. Comparative genomics of the major parasitic worms. Nat Genet. 2019;51(1):163-74.

11. Wang J, Gao S, Mostovoy Y, Kang Y, Zagoskin M, Sun Y, Zhang B, White LK, Easton A, Nutman TB, et al. Comparative genome analysis of programmed DNA elimination in nematodes. Genome Res. 2017;27(12):2001-14.

12. Jex AR, Liu S, Li B, Young ND, Hall RS, Li Y, Yang L, Zeng N, Xu X, Xiong Z, et al. Ascaris suum draft genome. Nature. 2011;479(7374):529-33.

13. Zhu XQ, Korhonen PK, Cai H, Young ND, Nejsum P, von SamsonHimmelstjerna G, Boag PR, Tan P, Li Q, Min J, et al. Genetic blueprint of the zoonotic pathogen Toxocara canis. Nat Commun. 2015;6:6145.

14. Schwarz EM, Hu Y, Antoshechkin I, Miller MM, Sternberg PW, Aroian RV. The genome and transcriptome of the zoonotic hookworm Ancylostoma 
ceylanicum identify infection-specific gene families. Nat Genet. 2015;47(4): 416-22.

15. Tang YT, Gao X, Rosa BA, Abubucker S, Hallsworth-Pepin K, Martin J, Tyagi R, Heizer E, Zhang X, Bhonagiri-Palsikar V, et al. Genome of the human hookworm Necator americanus. Nat Genet. 2014;46(3):261-9.

16. Foth BJ, Tsai IJ, Reid AJ, Bancroft AJ, Nichol S, Tracey A, Holroyd N, Cotton $J A$, Stanley EJ, Zarowiecki M, et al. Whipworm genome and dual-species transcriptome analyses provide molecular insights into an intimate hostparasite interaction. Nat Genet. 2014;46(7):693-700.

17. Jex AR, Nejsum P, Schwarz EM, Hu L, Young ND, Hall RS, Korhonen PK, Liao S, Thamsborg S, Xia J, et al. Genome and transcriptome of the porcine whipworm Trichuris suis. Nat Genet. 2014;46(7):701-6.

18. Godel C, Kumar S, Koutsovoulos G, Ludin P, Nilsson D, Comandatore F, Wrobel N, Thompson M, Schmid CD, Goto S, et al. The genome of the heartworm, Dirofilaria immitis, reveals drug and vaccine targets. FASEB J. 2012;26(11):4650-61.

19. Desjardins CA, Cerqueira GC, Goldberg JM, Dunning Hotopp JC, Haas BJ, Zucker J, Ribeiro JM, Saif S, Levin JZ, Fan L, et al. Genomics of Loa loa, a Wolbachia-free filarial parasite of humans. Nat Genet. 2013;45(5):495-500.

20. Lau YL, Lee WC, Xia J, Zhang G, Razali R, Anwar A, Fong MY: Draft genome of Brugia pahangi: high similarity between B. pahangi and B. malayi. Parasit Vectors 2015, 8:451.

21. Cotton JA, Bennuru S, Grote A, Harsha B, Tracey A, Beech R, Doyle SR, Dunn M, Hotopp JC, Holroyd N, et al. The genome of Onchocerca volvulus, agent of river blindness. Nat Microbiol. 2016;2:16216.

22. Xu L, Xu M, Sun X, Xu J, Zeng X, Shan D, Yuan D, He P, He W, Yang Y, et al. The genetic basis of adaptive evolution in parasitic environment from the Angiostrongylus cantonensis genome. PLoS Negl Trop Dis. 2019;13(11):e0007846.

23. Hunt VL, Tsai IJ, Coghlan A, Reid AJ, Holroyd N, Foth BJ, Tracey A, Cotton JA, Stanley EJ, Beasley H, et al. The genomic basis of parasitism in the Strongyloides clade of nematodes. Nat Genet. 2016;48(3):299-307.

24. Dillman AR, Macchietto M, Porter CF, Rogers A, Williams B, Antoshechkin I, Lee MM, Goodwin Z, Lu X, Lewis EE, et al. Comparative genomics of Steinernema reveals deeply conserved gene regulatory networks. Genome Biol. 2015;16:200.

25. Schiffer PH, Kroiher M, Kraus C, Koutsovoulos GD, Kumar S, Camps Jl, Nsah NA, Stappert D, Morris K, Heger P, et al. The genome of Romanomermis culicivorax: revealing fundamental changes in the core developmental genetic toolkit in Nematoda. BMC Genomics. 2013;14:923.

26. Mitreva M, Jasmer DP, Zarlenga DS, Wang Z, Abubucker S, Martin J, Taylor CM, Yin Y, Fulton L, Minx P, et al. The draft genome of the parasitic nematode Trichinella spiralis. Nat Genet. 2011;43(3):228-35.

27. Abad P, Gouzy J, Aury JM, Castagnone-Sereno P, Danchin EG, Deleury E, Perfus-Barbeoch L, Anthouard V, Artiguenave F, Blok VC, et al. Genome sequence of the metazoan plant-parasitic nematode Meloidogyne incognita. Nat Biotechnol. 2008;26(8):909-15.

28. Opperman CH, Bird DM, Williamson VM, Rokhsar DS, Burke M, Cohn J, Cromer J, Diener S, Gajan J, Graham S, et al. Sequence and genetic map of Meloidogyne hapla: a compact nematode genome for plant parasitism. Proc Natl Acad Sci U S A. 2008;105(39):14802-7.

29. Kikuchi T, Cotton JA, Dalzell JJ, Hasegawa K, Kanzaki N, McVeigh P, Takanashi T, Tsai IJ, Assefa SA, Cock PJ, et al. Genomic insights into the origin of parasitism in the emerging plant pathogen Bursaphelenchus xylophilus. PLoS Pathog. 2011;7(9):e1002219.

30. Eves-van den Akker S, Laetsch DR, Thorpe P, Lilley CJ, Danchin EG, Da Rocha M, Rancurel C, Holroyd NE, Cotton JA, Szitenberg A et al: The genome of the yellow potato cyst nematode, Globodera rostochiensis, reveals insights into the basis of parasitism and virulence. Genome Biol 2016, 17(1):124.

31. Cotton JA, Lilley CJ, Jones LM, Kikuchi T, Reid AJ, Thorpe P, Tsai IJ, Beasley H, Blok V, Cock PJ, et al. The genome and life-stage specific transcriptomes of Globodera pallida elucidate key aspects of plant parasitism by a cyst nematode. Genome Biol. 2014;15(3):R43.

32. Zheng J, Peng D, Chen L, Liu H, Chen F, Xu M, Ju S, Ruan L, Sun M. The Ditylenchus destructor genome provides new insights into the evolution of plant parasitic nematodes. Proc Biol Sci. 2016;283(1835):20160942.

33. McNulty SN, Strube C, Rosa BA, Martin JC, Tyagi R, Choi YJ, Wang Q, Hallsworth Pepin K, Zhang X, Ozersky P, et al. Dictyocaulus viviparus genome, variome and transcriptome elucidate lungworm biology and support future intervention. Sci Rep. 2016;6:20316.

34. Eccles D, Chandler J, Camberis M, Henrissat B, Koren S, Le Gros G, Ewbank $\mathrm{J}$. De novo assembly of the complex genome of Nippostrongylus brasiliensis using MinlON long reads. BMC Biol. 2018;16(1):6.
35. Laing R, Kikuchi T, Martinelli A, Tsai IJ, Beech RN, Redman E, Holroyd N, Bartley DJ, Beasley H, Britton C, et al. The genome and transcriptome of Haemonchus contortus, a key model parasite for drug and vaccine discovery Genome Biol. 2013;14(8):R88.

36. Weinstein DJ, Allen SE, Lau MCY, Erasmus M, Asalone KC, Walters-Conte K, Deikus G, Sebra R, Borgonie G, van Heerden E, et al. The genome of a subterrestrial nematode reveals adaptations to heat. Nat Commun. 2019; 10(1):5268.

37. Henkle-Duhrsen K, Tuan RS, Wildenburg G, Eschbach ML, Tawe W, Zipfel P, Walter RD. Localization and functional analysis of the cytosolic and extracellular CuZn superoxide dismutases in the human parasitic nematode Onchocerca volvulus. Mol Biochem Parasitol. 1997;88(1-2):187-202.

38. Lattemann CT, Matzen A, Apfel H. Up-regulation of extracellular copper/zinc superoxide dismutase mRNA after transmission of the filarial parasite Acanthocheilonema viteae in the vertebrate host Meriones unguiculatus. Int J Parasitol. 1999;29(9):1437-46.

39. Dabir S, Dabir P, Goswami K, Reddy MV. Prophylactic evaluation of recombinant extracellular superoxide dismutase of Brugia malayi in jird model. Vaccine. 2008;26(29-30):3705-10.

40. Baskaran P, Jaleta TG, Streit A, Rodelsperger C. Duplications and positive selection drive the evolution of parasitism-associated gene families in the nematode Strongyloides papillosus. Genome Biol Evol. 2017;9(3):790-801.

41. Stoltzfus JD, Minot S, Berriman M, Nolan TJ, Lok JB. RNAseq analysis of the parasitic nematode Strongyloides stercoralis reveals divergent regulation of canonical dauer pathways. PLoS Negl Trop Dis. 2012;6(10):e1854.

42. Soblik H, Younis AE, Mitreva M, Renard BY, Kirchner M, Geisinger F, Steen H, Brattig NW. Life cycle stage-resolved proteomic analysis of the excretome/ secretome from Strongyloides ratti-identification of stage-specific proteases. Mol Cell Proteomics. 2011;10(12):M111.010157.

43. Koutsovoulos GD, Poullet M, Elashry A, Kozlowski DKL, Sallet E, Da Rocha M, Perfus-Barbeoch L, Martin-Jimenez C, Frey JE, Ahrens $\mathrm{CH}$ et al: Genome assembly and annotation of Meloidogyne enterolobii, an emerging parthenogenetic root-knot nematode. Sci Data 2020;7(1):324.

44. Lian Y, Wei H, Wang J, Lei C, Li H, Li J, Wu Y, Wang S, Zhang H, Wang T, et al. Chromosome-level reference genome of X12, a highly virulent race of the soybean cyst nematode Heterodera glycines. Mol Ecol Resour. 2019;19(6): $1637-46$.

45. Sato K, Kadota Y, Gan P, Bino T, Uehara T, Yamaguchi K, Ichihashi Y, Maki N, Iwahori H, Suzuki T et al: High-Quality Genome Sequence of the Root-Knot Nematode Meloidogyne arenaria Genotype A2-O. Genome Announc 2018;6(26).

46. Somvanshi VS, Tathode M, Shukla RN, Rao U. Nematode genome announcement: a draft genome for rice root-knot nematode, Meloidogyne graminicola. J Nematol. 2018;50(2):111-6.

47. Lunt DH, Kumar S, Koutsovoulos G, Blaxter ML. The complex hybrid origins of the root knot nematodes revealed through comparative genomics. PeerJ. 2014;2:e356.

48. Szitenberg A, Salazar-Jaramillo L, Blok VC, Laetsch DR, Joseph S, Williamson VM, Blaxter ML, Lunt DH. Comparative genomics of apomictic root-knot nematodes: hybridization, ploidy, and dynamic genome change. Genome Biol Evol. 2017;9(10):2844-61.

49. Choi I, Subramanian P, Shim D, Oh BJ, Hahn BS. RNA-Seq of plant-parasitic nematode Meloidogyne incognita at various stages of its development. Front Genet. 2017:8:190.

50. Tanaka SE, Dayi M, Maeda Y, Tsai IJ, Tanaka R, Bligh M, Takeuchi-Kaneko Y, Fukuda K, Kanzaki N, Kikuchi T. Stage-specific transcriptome of Bursaphelenchus xylophilus reveals temporal regulation of effector genes and roles of the dauer-like stages in the lifecycle. Sci Rep. 2019;9(1):6080.

51. Vicente CS, Ikuyo Y, Shinya R, Mota M, Hasegawa K. Catalases induction in high virulence pinewood nematode Bursaphelenchus xylophilus under hydrogen peroxide-induced stress. PLoS One. 2015;10(4):e0123839.

52. Toppo S, Vanin S, Bosello V, Tosatto SC. Evolutionary and structural insights into the multifaceted glutathione peroxidase (Gpx) superfamily. Antioxid Redox Signal. 2008;10(9):1501-14.

53. Margis R, Dunand C, Teixeira FK, Margis-Pinheiro M. Glutathione peroxidase family - an evolutionary overview. FEBS J. 2008;275(15):3959-70.

54. Rhee SG, Woo HA, Kil IS, Bae SH. Peroxiredoxin functions as a peroxidase and a regulator and sensor of local peroxides. J Biol Chem. 2012;287(7): 4403-10.

55. Olahova M, Veal EA. A peroxiredoxin, PRDX-2, is required for insulin secretion and insulin/IIS-dependent regulation of stress resistance and longevity. Aging Cell. 2015;14(4):558-68. 
56. Kumar S, Stecher G, Suleski M, Hedges SB. TimeTree: a resource for timelines, timetrees, and divergence times. Mol Biol Evol. 2017;34(7):1812-9.

57. Fradin H, Kiontke K, Zegar C, Gutwein M, Lucas J, Kovtun M, Corcoran DL, Baugh LR, Fitch DHA, Piano F, et al. Genome architecture and evolution of a unichromosomal asexual nematode. Curr Biol. 2017;27(19):2928-39 e6.

58. Hiraki H, Kagoshima H, Kraus C, Schiffer PH, Ueta Y, Kroiher M, Schierenberg E, Kohara Y. Genome analysis of Diploscapter coronatus: insights into molecular peculiarities of a nematode with parthenogenetic reproduction. BMC Genomics. 2017;18(1):478

59. Chabaud AG, Bain O. The evolutionary expansion of the Spirurida. Int J Parasitol. 1994;24(8):1179-201.

60. Zelko IN, Mariani TJ, Folz RJ. Superoxide dismutase multigene family: a comparison of the CuZn-SOD (SOD1), Mn-SOD (SOD2), and EC-SOD (SOD3) gene structures, evolution, and expression. Free Radic Biol Med. 2002;33(3): 337-49.

61. Robinett NG, Peterson RL, Culotta VC. Eukaryotic copper-only superoxide dismutases (SODs): a new class of SOD enzymes and SOD-like protein domains. J Biol Chem. 2018;293(13):4636-43.

62. Xie H, Yuan D, Luo S, Zeng X, Zeng X, He P, Lv Z, Wu Z. Angiostrongylus cantonensis: an optimized cultivation of this parasitic nematode under laboratory conditions. Parasitol Res. 2017:116(8):2231-7.

63. Yuan D, Luo S, Xu L, Zeng X, Wu Z. Regulatory effect of host miR-101b-3p on parasitism of nematode Angiostrongylus cantonensis via superoxide dismutase 3. Biochimica et biophysica acta Gene regulatory mechanisms. 2019;1862(5):557-66.

64. Ou X, Tang L, McCrossan M, Henkle-Duhrsen K, Selkirk ME. Brugia malayi: localisation and differential expression of extracellular and cytoplasmic CuZn superoxide dismutases in adults and microfilariae. Exp Parasitol. 1995;80(3): 515-29.

65. Morassutti AL, Pinto PM, Dutra BK, Oliveira GT, Ferreira HB, Graeff-Teixeira C. Detection of anti-oxidant enzymatic activities and purification of glutathione transferases from Angiostrongylus cantonensis. Exp Parasitol. 2011;127(2):365-9.

66. Bienert GP, Schjoerring JK, Jahn TP. Membrane transport of hydrogen peroxide. Biochim Biophys Acta. 2006;1758(8):994-1003.

67. Han D, Antunes F, Canali R, Rettori D, Cadenas E. Voltage-dependent anion channels control the release of the superoxide anion from mitochondria to cytosol. J Biol Chem. 2003;278(8):5557-63.

68. Howe KL, Bolt BJ, Shafie M, Kersey P, Berriman M. WormBase ParaSite - a comprehensive resource for helminth genomics. Mol Biochem Parasitol. 2017;215:2-10

69. Lin $Q$, Fan S, Zhang $Y, X u M$, Zhang $H$, Yang $Y$, Lee AP, Woltering JM, Ravi V Gunter HM, et al. The seahorse genome and the evolution of its specialized morphology. Nature. 2016;540(7633):395-9.

70. Altschul SF, Gish W, Miller W, Myers EW, Lipman DJ. Basic local alignment search tool. J Mol Biol. 1990;215(3):403-10

71. Li R, Fan W, Tian G, Zhu H, He L, Cai J, Huang Q, Cai Q, Li B, Bai Y, et al. The sequence and de novo assembly of the giant panda genome. Nature. 2010; 463(7279):311-7.

72. Birney E, Clamp M, Durbin R. GeneWise and genomewise. Genome Res. 2004:14(5):988-95.

73. Eddy SR. A new generation of homology search tools based on probabilistic inference. Genome Inform. 2009:23(1):205-11.

74. Kall L, Krogh A, Sonnhammer EL. A combined transmembrane topology and signal peptide prediction method. J Mol Biol. 2004:338(5):1027-36.

75. Waterhouse AM, Procter JB, Martin DM, Clamp M, Barton GJ. Jalview version 2--a multiple sequence alignment editor and analysis workbench. Bioinformatics. 2009;25(9):1189-91.

76. Edgar RC. MUSCLE: multiple sequence alignment with high accuracy and high throughput. Nucleic Acids Res. 2004;32(5):1792-7.

77. Capella-Gutierrez S, Silla-Martinez JM, Gabaldon T. trimAl: a tool for automated alignment trimming in large-scale phylogenetic analyses. Bioinformatics. 2009;25(15):1972-3.

78. Nguyen LT, Schmidt HA, von Haeseler A, Minh BQ. IQ-TREE: a fast and effective stochastic algorithm for estimating maximum-likelihood phylogenies. Mol Biol Evol. 2015;32(1):268-74.

79. He Z, Zhang H, Gao S, Lercher MJ, Chen WH, Hu S. Evolview v2: an online visualization and management tool for customized and annotated phylogenetic trees. Nucleic Acids Res. 2016;44(W1):W236-41.

80. Letunic I, Bork P. Interactive Tree Of Life (iTOL) v4: recent updates and new developments. Nucleic Acids Res. 2019;47(W1):W256-9.
81. Li L, Stoeckert CJ Jr, Roos DS. OrthoMCL: identification of ortholog groups for eukaryotic genomes. Genome Res. 2003:13(9):2178-89.

82. Yang Z. PAML 4: phylogenetic analysis by maximum likelihood. Mol Biol Evol. 2007:24(8):1586-91.

83. Wang $Y$, Tang H, Debarry JD, Tan X, Li J, Wang X, Lee TH, Jin H, Marler B, Guo $H$, et al. MCScanX: a toolkit for detection and evolutionary analysis of gene synteny and collinearity. Nucleic Acids Res. 2012;40(7):e49.

84. Simão FA, Waterhouse RM, loannidis P, Kriventseva EV, Zdobnov EM. BUSCO: assessing genome assembly and annotation completeness with single-copy orthologs. Bioinformatics. 2015;31(19):3210-2.

85. Bolger AM, Lohse M, Usadel B. Trimmomatic: a flexible trimmer for Illumina sequence data. Bioinformatics. 2014;30(15):2114-20.

86. Kim D, Langmead B, Salzberg SL. HISAT: a fast spliced aligner with low memory requirements. Nat Methods. 2015;12(4):357-60.

87. Liao Y, Smyth GK, Shi W. featureCounts: an efficient general purpose program for assigning sequence reads to genomic features. Bioinformatics. 2014;30(7):923-30

88. Yanai I, Benjamin H, Shmoish M, Chalifa-Caspi V, Shklar M, Ophir R, Bar-Even A, Horn-Saban S, Safran M, Domany E, et al. Genome-wide midrange transcription profiles reveal expression level relationships in human tissue specification. Bioinformatics. 2005;21(5):650-9.

89. Feng J, Meyer CA, Wang Q, Liu JS, Shirley Liu X, Zhang Y. GFOLD: a generalized fold change for ranking differentially expressed genes from RNA-seq data. Bioinformatics. 2012:28(21):2782-8.

\section{Publisher's Note}

Springer Nature remains neutral with regard to jurisdictional claims in published maps and institutional affiliations.

\section{Ready to submit your research? Choose BMC and benefit from:}

- fast, convenient online submission

- thorough peer review by experienced researchers in your field

- rapid publication on acceptance

- support for research data, including large and complex data types

- gold Open Access which fosters wider collaboration and increased citations

- maximum visibility for your research: over $100 \mathrm{M}$ website views per year

At $\mathrm{BMC}$, research is always in progress.

Learn more biomedcentral.com/submissions 